\title{
Retailer's Optimal Procurement Strategy under Supply Disruption and Stochastic Demand: Backup Sourcing or Simultaneous Sourcing
}

\author{
Feng Lin $\mathbb{D}^{1},{ }^{1}$ Jinzhao Shi $\mathbb{D},{ }^{2}$ Peng $W u \mathbb{D},{ }^{1}$ and Xingxuan Zhuo ${ }^{1}$ \\ ${ }^{1}$ School of Economics and Management, Fuzhou University, Fuzhou, China \\ ${ }^{2}$ School of Economics and Management, Chang'an University, Xi'an, China \\ Correspondence should be addressed to Peng Wu; wupeng88857@gmail.com
}

Received 14 February 2020; Revised 6 June 2020; Accepted 25 June 2020; Published 28 August 2020

Academic Editor: Toshikazu Kuniya

Copyright (C) 2020 Feng Lin et al. This is an open access article distributed under the Creative Commons Attribution License, which permits unrestricted use, distribution, and reproduction in any medium, provided the original work is properly cited.

Practically, supply disruption may lead production process to entirely halt (completely disrupted) or the output to differ in the order size (partially disrupted), which makes it more difficult for the retailer to satisfy stochastic market demand. Under the circumstance, the retailer is likely to procure products from two suppliers to effectively alleviate the demandsupply mismatches. Thus, under supply disruption and stochastic demand, this paper develops both backup sourcing and simultaneous sourcing (SS) strategies to analyze the retailer's performance, where backup sourcing includes wholesale price priority (WPP) and supply reliability priority (SRP). Specifically, (1) under WPP, when the selling price is relatively lower (higher), the retailer is suggested to activate the reliable backup supplier after the realization of supply disruption (demand uncertainty). (2) Under SRP, two scenarios including minor disruption and major disruption can be identified, where the retailer's order quantity from the reliable (unreliable) supplier under minor disruption scenario is more (less) than that under major. (3) Finally, this paper systematically compares the retailer's preferences among WPP, SRP, and SS via theoretical results and numerical examples. That is, when the unreliable supplier is more likely to work normally or shortage cost (selling price) is relatively lower, the retailer prefers SPR regarding the unreliable supplier as backup sourcing due to its lower wholesale price and acceptable supply disruption. Otherwise, the retailer is inclined to WPP regarding the reliable supplier as backup sourcing for ensuring all market demand to be satisfied. In addition, unless the emergency prices of two suppliers are extremely higher, backup sourcing strategies could perform better than simultaneous sourcing strategy.

\section{Introduction}

In practice, the upstream supply process is highly vulnerable to supply disruption, ranging from major breakdowns because of natural or man-made disasters (e.g., earthquakes and floods) to minor interruptions due to material contamination and labour problems $[1,2]$. As a result, the supply process may entirely halt (completely shut down) or the output differs in the order size (partially disrupted), leading to supply shortage, loss of goodwill, and the collapse of supply chain, which has already been viewed as one of the major challenges to supply chains success and caused massive economic consequences $[3,4]$. For example, the volcanic eruption in Iceland in March 2010 caused the air cargo transportation to be completely paralyzed. Therefore, many companies with production plants in Europe (such as BMW, Nissan, and Audi) had to suspend production and shut down the factory due to the supply interruption of key parts [5]. Further, as Silbermayr and Minner [6] noted, according to a survey of 519 respondents from 71 countries across 15 industry sectors in 2015, 75\% of the respondents experienced at least one supply chain disruption. Further, from the downstream side, the supply chain is also confronted with demand risk due to the variation of market demand, which further complicates the matches between supply and demand [7]. Under the circumstance, the retailer 
is subject to the combined negative effects of supply disruption and stochastic demand, which may significantly lower operational performances.

To mitigate negative effects of stock-out risk, it is gradually acknowledged that the retailer could adopt backup sourcing strategy for hedging against supply disruption and stochastic demand, which has been widely adopted in aircraft, pharmaceutical, and electronic industries [8]. That is, two suppliers provide products with similar quality but differ in supply uncertainty and selling price, where one supplier is viewed as major source and the other is taken as backup sourcing which would be initiated after observing the major supplier's supply [1]. This procurement strategy is able to prevent the retailer from potential supply disruption and simultaneously control operating costs, which enhances the flexibility and stability of the supply chain [9]. Taking an example of Japan tsunami on 2011 March, Toyota North America only operated its $30 \%$ capacity due to the lack of part supply from Japan, while General Motors successfully solved the supply shortages of 118 parts by implementing backup plans [10]. Generally, under backup sourcing strategy, the retailer could procure products from major sourcing under demand uncertainty, and the backup sourcing can be postponed after market demand or supply disruption is realized. Thus, under supply disruption and stochastic demand, there are two types of backup sourcing strategies based on the retailer's order sequences $[1,3]$.

One of the typical forms is wholesale price priority (WPP) strategy, where the unreliable supplier with lower wholesale price is regarded as the major sourcing, and the reliable supplier can be treated as the backup supplier. Further, the reliable backup supplier can be activated after the supply disruption occurs or both supply and demand risks are realized, which can be labeled as WPP-SR and WPP-DR strategy, respectively. Another typical form is supply reliability priority (SRP) strategy, where the reliable supplier with higher wholesale price is viewed as major sourcing, while the unreliable supplier with lower wholesale price is viewed as backup supplier.

To sum up, the backup sourcing strategy can be viewed as emergency procurement requiring higher emergency cost, which mitigates the high loss due to the occurrence of supply disruption [5]. Although WPP and SRP have found their wide applications to prevent the retailer from stock risk, it is urgent for the retailer to identify the most appropriate backup sourcing strategy via trading off supply disruption and stochastic demand as well as wholesale prices.

In addition to backup sourcing, simultaneous sourcing (SS) strategy from two suppliers is another effective policy for the retailer to hedge against supply disruption. That is, when market demand is stochastic, the retailer procures products from both the unreliable supplier and the reliable supplier at the same time. Under this policy, the retailer could take the advantage of lower wholesale price from two suppliers and avoid expensive emergency costs, while it is responsible for demand risk. To summarize, under supply disruption and stochastic demand, the following three urgent issues are required to be further analyzed:
(1) Under WPP, what are the differences between activating backup reliable supplier after supply disruption and for demand uncertainty?

(2) Under SRP, how does supply disruption of the unreliable supplier impact the retailer's procurement decisions from major and backup suppliers?

(3) When involving both backup sourcing (including WPP and SRP) and simultaneous sourcing strategies, what is the retailer's optimal sourcing strategy under supply disruption and stochastic demand?

To solve the previous issues, in this paper, the retailer is assumed to procure products from two suppliers for hedging against supply disruption and stochastic demand. And different backup sourcing and simultaneous sourcing strategies are involved to address the retailer's optimal procurement policy. And the major contributions of this paper can be summarized as follows:

(1) Firstly, two forms of WPP strategies are further classified on the basis of the reliable supplier to be initiated after the occurrence of supply disruption (i.e., WPP-SR) or the realization of both supply and demand uncertainties (i.e., WPP-DR). Specifically, WPP-SR ensures the retailer's order quantity from unreliable supplier to be always met, while WPP-DR guarantees the market demand to be entirely satisfied with more expensive emergency cost. Further, positive relationships between selling price (shortage cost) and the retailer's order quantity from the reliable supplier can be identified under WPP-SR, which do not exist under WPP-DR. In addition, when the procurement or emergency price of the reliable supplier is relatively higher, the retailer's order quantity from the reliable supplier under WPP-SR is less than that of WPP-DR.

(2) Secondly, under SRP, it is surprising that the retailer's optimal procurement solutions from two suppliers consist of two scenarios including minor disruption and major disruption. Specifically, (i) under minor disruption scenario, the unreliable supplier is more likely to supply normally or offer a higher value of emergency price, implying that the retailer would assume a higher-order quantity from the reliable supplier when demand is uncertain. And after the realization of the demand, the retailer accurately places order quantity from the unreliable supplier based on the difference between the supply of the reliable sourcing and the demand. (ii) Under major disruption scenario, on the contrary, the retailer would decrease the order quantity from the reliable supplier (compared with that under minor disruption scenario) and set the order quantity from the unreliable supplier to exceed the gap between the reliable supplier's products and the realized demand.

(3) Finally, under supply disruption, this paper systematically compares the retailer's preferences towards backup sourcing and simultaneous sourcing strategies 
via theoretical results and numerical examples. (i) Since WPP-DR could ensure market demand to be entirely satisfied by expensive emergency replenishment from the reliable supplier, the retailer would regard it as the optimal backup sourcing when selling price or shortage cost is relatively higher. On the contrary, the retailer would adopt SRP for directly improving the supply reliability and avoiding expensive emergency replenishment from the reliable supplier. (ii) When the unreliable supplier is less likely to work normally, the retailer would prefer WPP-DR and regard the reliable supplier as backup sourcing for ensuring all market demand to be satisfied. Otherwise, the retailer would prefer SPR regarding the reliable supplier as backup sourcing due to its lower wholesale price and its acceptable supply disruption. (iii) In addition, unless the emergency prices of two suppliers are extremely higher, backup sourcing strategies could perform better than simultaneous sourcing strategies.

The remainder of this paper is organized as follows. Section 2 presents a series of literature reviews concerning supply disruption, backup sourcing, and simultaneous sourcing strategies. And Section 3 depicts key assumptions and relevant notations which are used to present the models. Then, Sections 4-6 take views of WPP, SRP, and SS to investigate the retailer's optimal procurement policies, respectively. Furthermore, in Section 7, several numerical examples are provided to explicitly derive more managerial insights. Finally, derived results are summarized in Section 8.

\section{Literature Review}

In view of negative impacts of supply disruption towards operational practices, a series of researches have been conducted to optimize operational performance of firms. Schmitt et al. [11] integrated discrete-event uncertainty (disruptions) and continuous sources of uncertainty to address their effects on optimal solutions for newsvendor models. Under the deterministic demand setting, Tang et al. [12] assumed the buyer to offer incentives (including investment subsidy and inflated order quantity) to the supplier under supply disruption for enhancing process reliability. Under supply and demand uncertainty, Ray and Jenamani [13] applied newsvendor approach to formulate two models for optimal order allocation of a risk-neutral or risk-averse decision-maker. Inderfurth and Kiesmuller [14] established a periodic-review inventory model for a single item, which involves two different types of uncertain supply, including binomial and stochastically proportional yield. Nevertheless, besides the all-or-nothing setting (i.e., binomial) concerning supply disruption, several scholars have pointed out that supply disruption may also lead to partial disruption, where the manufacturer's final output could be a fraction between complete shutdown and working normally. Based on this assumption, Hou et al. [1] applied capacity reservation and minimum order quantity to mitigate the disruption risk, while Zeng and Xia [10] adopted a decision-tree approach to consider disruption situations at all levels.
To hedge against supply disruption, a series of researches have incorporated wholesale price priority (WPP), the most widely adopted backup sourcing strategy, to improve supply reliability under the deterministic demand setting. Yin and Wang [15] proposed three common options including advance purchase, reservation, and contingency purchase for firms to adopt when cooperating with the backup supplier to hedge against the disruption risk. Via a sequential optimization process, Hou et al. [16] applied a buy-back contract to coordinate a buyer with a backup supplier, when the main supplier experiences supply disruption. In a continuousreview inventory problem, Qi [17] used the order-up-to policy to explore the retailer's operational solutions when the primary supplier is subject to random disruptions.

Further, in the context of WPP, several studies have also analyzed the retailer's procurement policy under supply disruption and stochastic demand. Giri and Bardhan [5] built a two-echelon supply chain under uncertain demand and supply disruption, where the penalty contract mechanism is applied to achieve supply chain coordination. Zhang et al. [4] adopted a procurement strategy with backup sourcing under stochastic supply and demand, where the game-theoretic framework is involved to explore optimal decisions of the players. Nevertheless, it can be concluded that these papers simply assume backup supplier to be initiated after supply disruption is realized, which do not distinguish WPP-SR from WPP-DR. Additionally, in their studies, partial disruption is excluded from supply disruption, which certainly merits additional attention.

In addition to backup sourcing, simultaneous sourcing from two suppliers is another effective policy to hedge against supply disruption. Under supply disruption, Silbermayr and Minner [6] compared risk reduction via dual sourcing with learning effects when negotiating with a single supplier via a long-term contract. Under disruption risk, Kumar, Basu, and Avittathur [9] analyzed the retailer's optimal pricing and dual-sourcing policies for competing with another retailer supplied by a reliable supply chain. In a two-stage supply chain with a nonstationary and pricesensitive deterministic demand, Yu et al. [18] quantified impacts of supply disruption towards single and dual sourcing. Nevertheless, existing researches are still lack of addressing simultaneous sourcing policy under partial supply disruption and demand uncertainty.

To our best knowledge, Li [3] is the closest to our study, which systematically addresses the retailer's procurement policy from two unreliable suppliers under deterministic demand. Specifically, Li [3] incorporated both the all-ornothing supply disruption and the proportional random yield to analyze how supply risks influence the retailer's order decisions. Further, under deterministic demand, Li [3] compared different decision sequences including backup sourcing and simultaneous sourcing strategies, suggesting the retailer to regard the relatively expensive supplier as a backup source via computational experiments. Unlike Li [3], this paper desires to incorporate both stochastic demand and supply disruption to vary decision sequences to generate more procurement strategies. It indicates that the retailer could place orders from two suppliers before or after 
demand uncertainty (or supply disruption), resulting in WPP-SR, WPP-DR, SRP, and SS. And compared with Li [3], due to the joint effects of stochastic demand and partial disruption, this paper identified that the retailer shows different order incentives and procurement preferences towards backup sourcing and simultaneous sourcing strategies.

To sum up, in order to explicitly distinguish this paper from previous studies, the following table is adopted to summarize key characteristics of the above-mentioned papers under supply disruption (Table 1).

\section{Model Description}

The following notations and assumptions are adopted to develop new proposed models throughout the paper.

\subsection{Notations}

$i$ : $i=1,2$ represent supplier 1 and supplier 2, respectively

$j: j=\mathrm{WS}, \mathrm{WD}, \mathrm{SR}$, and SS denote WPP-SR, WPP-DR, SRP, and SS, respectively

$p$ : the unit selling price of the retailer

$w_{i}$ : the unit procurement price of supplier $i$ when demand is stochastic

$\widetilde{w}_{i}$ : the unit emergency price of supplier $i$ when demand is deterministic

$c_{s}$ : the unit shortage cost of the retailer

$c_{o}$ : the unit holding cost of the retailer

Q: the retailer's order quantity from supplier 1 (decision variable)

$R$ : the retailer's order quantity from supplier 2 (decision variable)

$y$ : the disruption magnitude $(0 \leq y \leq 1)$, indicating the fraction from supplier 2

$m$ : the probability of supplier 2 to completely break down; that is, $y=0$

$n$ : the probability of supplier 2 to work normally; that is, $y=1$

$\Pi^{j}$ : the retailer's expected profit under $j$ strategy

\subsection{Assumptions}

(1) In a single period, this paper focuses on the retailer's procurement problem where two suppliers offer similar products. Supplier 1 is perfectly reliable with a higher wholesale price, while supplier 2 is subject to supply disruption with a lower wholesale price.

(2) At the beginning of the period (i.e., stage 1), the market demand $D$ is assumed to be stochastic, where the cumulative and probability distribution functions of $D$ are $F(D)$ and $f(D)$, respectively. And market demand $F(D)$ is differentiable, strictly increasing, while its complementary CDF $\bar{F}(D)=1-F(D)$. Further, at the end of the period (i.e., stage 2), the market demand $D$ can be realized, where the emergency replenishment can be proceeded via backup sourcing.

(3) The retailer's order quantities from suppliers 1 and 2 are $Q$ and $R$, respectively. At stage 1 when market demand is stochastic, wholesale prices of products of suppliers 1 and 2 are $w_{1}$ and $w_{2}$, respectively, where $p>w_{1}>w_{2}$. And at stage 2 after demand is revealed, wholesale prices of suppliers 1 and 2 would increase to $\widetilde{w}_{1}$ and $\widetilde{w}_{2}$, respectively, where $\widetilde{w}_{1}>\widetilde{w}_{2}$.

(4) Supplier 1 can fully fulfill the retailer's order quantity $Q$, while supplier 2 is subject to supply disruption, leading final output to be $y R$. By referring to Hou et al. [1] and Zeng and Xia [10], $y$ is a fraction between 0 and 1 representing the magnitude of a disruption, which can be

$$
g(y)= \begin{cases}m, & y=0, \\ 1-m-n, & 0<y<1, \\ n, & y=1 .\end{cases}
$$

From (1), the supplier 2's probability of entire shutdown $(y=0)$ and entire reliable $(y=1)$ is $m$ and $n$, respectively. And the probability of being partially disrupted is $1-m-n$, which is continuous for $0<y<1$. In addition, the retailer should pay off the purchase cost based on the received quantity as soon as receiving products from supplier 1 or supplier 2 .

(5) In this paper, the following two backup sourcing strategies are involved.

(i) Wholesale price priority (WPP): the retailer procures $R$ units from the unreliable supplier 2 with $w_{2}$ when demand is stochastic. Further, the reliable backup supplier would be ordered by the retailer with $w_{1}$ after the supply disruption occurs or with $\widetilde{w}_{1}$ after both supply and demand risks are realized, which can be labeled as WPP-SR and WPP-DR strategy, respectively.

(ii) Supply reliability priority (SRP): the retailer procures $Q$ units from the reliable supplier 1 with $w_{1}$ when market demand is uncertain. And the retailer conducts emergency procurement from the unreliable supplier 2 with $\widetilde{w}_{2}$ when market demand is realized.

(iii) Simultaneous sourcing (SS): when demand is stochastic, the retailer simultaneously procures $Q$ and $R$ units from the reliable supplier 1 and the unreliable supplier 2, respectively.

\section{Retailer's Optimal Procurement Policy under WPP}

As previously mentioned, there are two types of WPP on the basis of the emergency order from supplier 1 to be conducted after supply disruption is realized (WPP-SR) or both supply and demand are realized (WPP-DR). 
TABLE 1: Literature reviews of backup (simultaneous) sourcing models under supply disruption.

\begin{tabular}{|c|c|c|c|c|c|}
\hline \multirow{2}{*}{ Authors } & \multirow{2}{*}{ Supply disruption } & \multirow{2}{*}{ Demand } & \multicolumn{2}{|c|}{ Backup sourcing } & \multirow{2}{*}{ Simultaneous sourcing } \\
\hline & & & WPP & SRP & \\
\hline Hou et al. [1] & Partial & Det. & & & $\checkmark$ \\
\hline $\operatorname{Li}[3]$ & Binomial & Det. & WPP-SR & $\checkmark$ & $\checkmark$ \\
\hline Giri and Bardhan [5] & Binomial & Sto. & WPP-DR & & \\
\hline Silbermayr and Minner [6] & Binomial & Det. & & & $\checkmark$ \\
\hline Kumar et al. [9] & Binomial & Det. & & & $\checkmark$ \\
\hline Zeng and Xia [10] & Partial & Sto. & & & $\checkmark$ \\
\hline Tang et al. [12] & Binomial & Det. & & & $\checkmark$ \\
\hline Yin and Wang [15] & Binomial & Det. & WPP-SR & $\checkmark$ & \\
\hline Hou et al. [16] & Binomial & Det. & WPP-SR & & \\
\hline Qi [17] & Binomial & Det. & WPP-SR & & \\
\hline Zhang and Wang [19] & Binomial & Sto. & WPP-DR & & \\
\hline Yu et al. [18] & Binomial & Det. & WPP-SR & & $\checkmark$ \\
\hline Present paper & Partial & Sto. & $\begin{array}{l}\text { WPP-DR, } \\
\text { WPP-SR }\end{array}$ & $\checkmark$ & $\checkmark$ \\
\hline
\end{tabular}

Note. For convenience, let terms Det. and Sto. denote deterministic and stochastic, respectively.

4.1. Optimal Procurement Policy under WPP-SR. Under WPP-SR (see Figure 1(a)), the retailer should firstly order $R$ units with $w_{2}$ from the unreliable supplier 2 to satisfy the stochastic demand. And after supply disruption is revealed, the retailer receives $y R$ from the unreliable supplier 2 and then further procures $Q$ units (i.e., $R-y R$ ) with $w_{1}$ from supplier 1 to ensure that the amount of available products equals $R$ units. Therefore, considering the supply disruption of supplier 2 , the retailer's expected profit function can be presented as follows:

(1) When the supplier 2 supply process is entirely disrupted, we have $y R=0$ and the retailer would procure $R$ (i.e., $Q=R-y R$ ) units from supplier 1 with the wholesale price $w_{1}$. Under the circumstance, $\Pi_{11}^{W S}=E\left[p \min (D, R)-c_{s}\right.$ $\left.(D-R)^{+}-c_{o}(R-D)^{+}-w_{1} R\right]$.

(2) When the supplier's supply process is entirely normal, we have $y R=R$ and there is no need for the retailer to further order products from supplier 1 . Thus, $\Pi_{12}^{W S}=E\left[p \min (D, R)-c_{s}(D-R)^{+}-c_{o}(R-\right.$ $\left.D)^{+}-w_{2} R\right]$.

(3) When the supplier's supply process is partially disrupted, that is, $R>y R>0$, the retailer would procure the rest of the products from supplier 1 . Thus, we have $\quad \Pi_{13}^{W S}=E\left[p \min (D, R)-c_{s}(D-R)^{+}-\right.$ $\left.c_{o}(R-D)^{+}-w_{1}(1-y) R-w_{2} y R\right]$.

Hence, the retailer's expected profit function under WPP-SR can be depicted as follows:

$$
\Pi_{1}^{\mathrm{WS}}(R)=m \Pi_{11}^{\mathrm{WS}}+n \Pi_{12}^{\mathrm{WS}}+(1-m-n) \int_{0}^{1} \Pi_{13}^{\mathrm{WS}} \mathrm{d} y .
$$

According to (2), the following theorem can be summarized.

Theorem 1. Under WPP-SR, the retailer's optimal order quantity $R^{W S}$ from the unreliable supplier 2 can be uniquely obtained by the following expression: $\bar{F}\left(R^{W S}\right)=\Delta_{1}$, where $\Delta_{1}=\left[(1+m-n) w_{1}+(1+n-m) w_{2}+2 c_{o}\right] /\left[2\left(p+c_{s}+c_{o}\right)\right]$.
Proof. The details can be found at Appendix A. From Theorem 1 , it can be found that WPP-SR ensures that the retailer's order quantity $R^{\mathrm{WS}}$ from the unreliable supplier 2 can be always met by emergency replenishment from the reliable supplier 1 (i.e., $\left.(1-y) R^{\mathrm{WS}}\right)$ and enables the retailer to avoid higher emergency procurement price $\widetilde{w}_{1}$ of supplier 1 after both supply and demand uncertainty are realized. Furthermore, it is apparent that $R^{\mathrm{WS}}$ is positively related to $p$ and $c_{s}$, while it is negatively related to $w_{1}$ and $w_{2}$. That is, under WPP-SR, the retailer would increase (decrease) the order quantity from the unreliable supplier 2 when confronted with higher value of selling profit and shortage cost (procurement prices). In addition, since $w_{1}>w_{2}$, the retailer would place a higher-order quantity from the unreliable supplier 2 when supplier 2 is more (less) likely to work normally (complete shutdown).

4.2. Optimal Procurement Policy under WPP-DR. Under WPP-DR strategy, the decision-making process of the retailer's order allocation scheme consists of two stages (see Figure 1(b)). (1) In the first stage, when the market demand is stochastic, the retailer firstly orders $R$ units of the product from the unreliable supplier 2 with the lower price $w_{2}$. (2) In the second stage, the retailer has received the final output $y R$, and the market demand can be realized. Then, the retailer will decide whether to place an emergency order $Q$ from supplier 1 with $\widetilde{w}_{1}$. Thus, under WPP-DR strategy, the backward induction method can be used to obtain the retailer's optimal procurement policy.

4.2.1. Procurement Policy at the Second Stage. In the second stage, the market demand $D$ and the supplier 2's final output $y R$ are revealed to the retailer. Therefore, the retailer should order $Q$ units from supplier 1 on the basis of the difference between supply and demand in the first stage, that is, $D-y R$. In summary, the following lemma can be obtained.

Lemma 1. Under the second stage of WPP-DR, the retailer's optimal order quantity from supplier $1 Q^{W D}=(D-y R)^{+}$. 


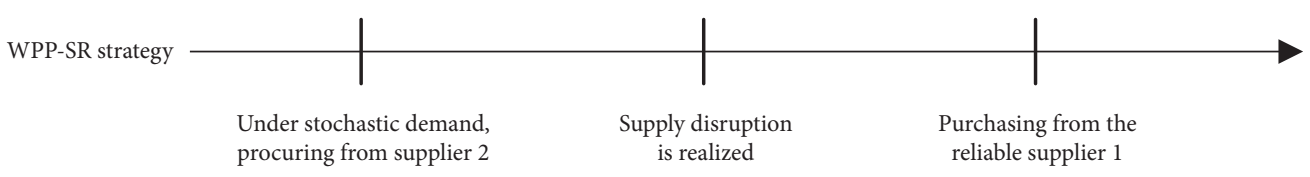

(a)

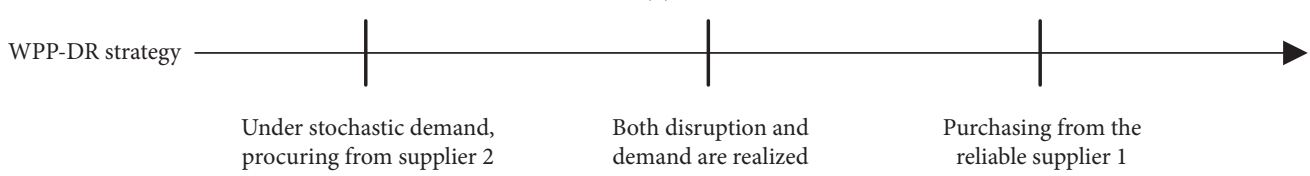

(b)

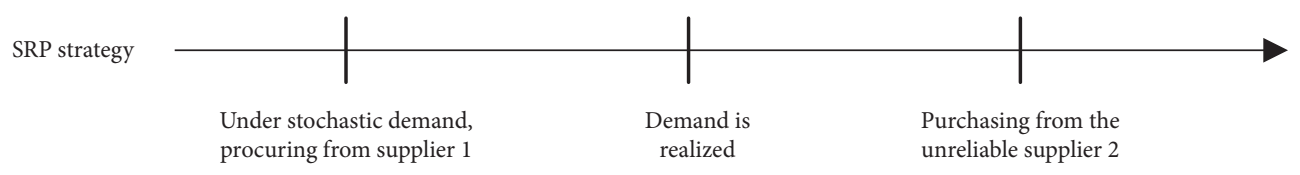

(c)

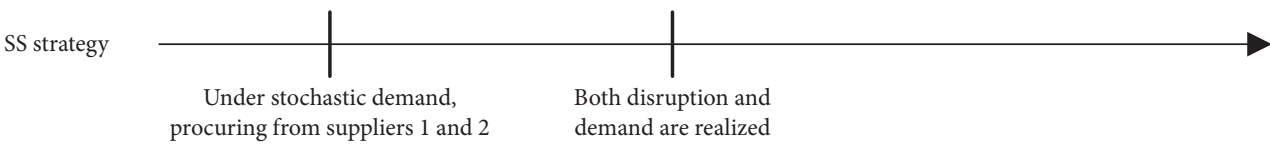

(d)

FiguRE 1: Retailer's order sequences under different backup and simultaneous sourcing strategies.

From Lemma 1, since the supplier 1's supply process is reliable, the market demand can be always satisfied under WPP-DR with higher emergency cost. Specifically, if $D<y R$, the retailer would not adopt emergency order; otherwise, the supplier 1 could fully deliver $D-y R$ units to the retailer.

4.2.2. Procurement Policy at the First Stage. In the first stage, market demand $D$ is stochastic and the retailer should order $R$ units from the unreliable supplier 2. Therefore, considering the supply disruption of supplier 2, the retailer's expected profit function can be presented as follows:

(1) When the supplier 2 supply process is entirely disrupted, we have $y R=0$ and the retailer would procure $D-R$ (i.e., $Q=R-y R$ ) units from supplier 1 with the wholesale price $\widetilde{w}_{1}$. Under the circumstance, $\Pi_{11}^{\mathrm{WD}}=E\left[\left(p-\widetilde{w}_{1}\right) D\right]$.

(2) When the supplier 2 supply process is entirely normal, we have $y R=R$. Then, if $R>D$, the retailer should pay off additional holding cost; otherwise, the retailer should further order $D-R$ units from supplier 1 . Thus, based on the stochastic demand $D$, $\Pi_{12}^{\mathrm{WD}}=\int_{0}^{R}\left[p D-c_{o}(R-D)\right] f(D) \mathrm{d} D+\int_{R}^{+\infty}$ $\left[p D-\widetilde{w}_{1}(D-R)\right] f(D) \mathrm{d} D-w_{2} R$.

(3) When the supplier 2 supply process is partially disrupted, that is, $R>y R>0$, then, the retailer should either pay off holding cost for the surplus products $D-y R$ or procure the shortages from supplier 1. Thus, we have $\Pi_{13}^{\mathrm{WD}}=\int_{0}^{y R}\left[p D-c_{o}(y R-\right.$ $D)] f(D) \mathrm{d} D+\int_{y R}^{+\infty}\left[p D-\widetilde{w}_{1}(D-y R)\right] f(D) \mathrm{d} D-$ $w_{2} y R$.

Hence, the retailer's expected profit function under WPP-DR can be depicted as follows:

$$
\Pi_{1}^{\mathrm{WD}}(R)=m \Pi_{11}^{\mathrm{WD}}+n \Pi_{12}^{\mathrm{WD}}+(1-m-n) \int_{0}^{1} \Pi_{13}^{\mathrm{WD}} \mathrm{d} y
$$

Based on (3), the following theoretical results can be summarized.

Theorem 2. Under WPP-DR, the retailer's optimal order quantity $R^{W D}$ from the unreliable supplier 2 can be uniquely obtained by the following expression: $n \bar{F}\left(R^{W D}\right)+(1-n-m)$ $\int_{0}^{1} y \bar{F}\left(y R^{W D}\right) d y=\left[(1+n-m)\left(c_{o}+w_{2}\right)\right] /\left[2\left(c_{o}+\widetilde{w}_{1}\right)\right]$.

Proof. The details can be found at Appendix B. From Theorem 2, WPP-DR ensures that the market demand can be always satisfied by the reliable backup sourcing with expensive emergency cost from supplier 1 with $\widetilde{w}_{1}$. Under the circumstance, the retailer's order quantity $R^{\mathrm{WD}}$ is irrelevant to $p$ and $c_{s}$, which is totally different from the results derived from WPP-SR. Further, the retailer would increase order quantity from the unreliable supplier 2 when confronted with higher (lower) values of $\widetilde{w}_{1}\left(w_{2}\right)$. And based on Theorems 1 and 2, the following theoretical results can be obtained.

Corollary 1. Let $\Delta_{2}=\left(c_{o}+w_{2}\right) /\left(c_{o}+\widetilde{w}_{1}\right)$ and $\Delta_{3}=[(1+$ $\left.n-m) w_{2}-(1-n-m) \widetilde{w}_{1}+2 n c_{o}\right] /\left[2 n\left(c_{o}+\widetilde{w}_{1}\right)\right]$, the following hold true:

(1) If $\Delta_{1} \geq \Delta_{2}$, then $R^{\mathrm{WS}} \leq R^{\mathrm{WD}}$,

(2) If $\Delta_{1}<\Delta_{3}$, then $R^{\mathrm{WS}}>R^{\mathrm{WD}}$.

Proof. The details can be found at Appendix C. From Corollary 1, the differences concerning the retailer's order 
quantity from sourcing reliable supplier 1 between WPP-SR and WPP-DR can be explicitly identified. Specifically, when $p$ or $c_{s}$ is relatively lower or $w_{1}\left(\widetilde{w}_{1}\right)$ is relatively higher, $\Delta_{1}$ is more likely to be greater than $\Delta_{2}$, implying that the retailer's order quantity $R^{\mathrm{WS}}$ from the unreliable supplier 2 under WPP-SR is less than that of WPP-DR. Otherwise, when $p$ or $c_{s}$ increases to some degree or $w_{1}\left(\widetilde{w}_{1}\right)$ is relatively lower, $\Delta_{1}$ may be less than $\Delta_{3}$, indicating that the retailer would order less quantities from the unreliable supplier 2 under WPPDR.

\section{Retailer's Optimal Procurement Policy under SRP}

In SRP strategy, the retailer's procurement decision process can be divided into two stages (see Figure 1(c)). Specifically, (1) in the first stage (at the beginning of the selling period), the retailer should order $Q$ units from the reliable supplier 1 at a higher price $w_{1}$ to meet the stochastic market demand. (2) In the second stage (at the end of selling period), the market demand can be revealed by the retailer. And based on the gap between the realized demand and the supplied quantity $Q$ by supplier 1 , the retailer can urgently order $R$ units from unreliable supplier 2 with a lower price $\widetilde{w}_{2}$. To sum up, the retailer optimal procurement policy under SRP can be solved by the backward induction method, which can be formulated as follows.

5.1. Optimal Procurement Policy at the Second Stage. In the second stage, the retailer is aware of both market demand $D$ and supplier 1's delivery quantity $Q$. Therefore, according to the differences between supply and demand in the first stage (i.e., $D-Q$ ), there are two situations to analyze:

(1) If $D \leq Q$, the supplied quantity of supplier 1 can effectively meet the market demand, indicating that the retailer will not order any product from the unreliable supplier 2 at this stage; that is, $R^{\mathrm{SR}}=0$.

(2) If $D>Q$, the retailer should urgently purchase $R$ units from the unreliable supplier 2. According to the probability density function $g(y)$, three situations can be obtained concerning the actual output $y R$.

(i) When supplier 2 is completely disrupted with the probability of $m(y R=0)$, the retailer's profit equals $\Pi_{21}^{\mathrm{SR}}=p Q-c_{s}(D-Q)^{+}$.

(ii) When supplier 2 fully supplies products with probability $n$ (i.e., $y R=R$ ), the retailer's profit equals $\Pi_{22}^{\mathrm{SR}}=p \min (D, Q+R)-c_{s}(D-Q-R)^{+}$ $-c_{o}(Q+R-D)^{+}-\widetilde{w}_{2} R$.

(iii) When supplier 2's product supply is partially disrupted (i.e., $R>y R>0$ ), the retailer's profit equals $\quad \Pi_{23}^{\mathrm{SR}}=p \min (D, Q+y R)-c_{s}$ $(D-Q-y R)^{+}-c_{o}(Q+y R-D)^{+}-\widetilde{w}_{2} y R$.

To sum up, in stage 2 , since the market demand is deterministic, in the context of $D>Q$, the retailer's expected profit function at the second stage can be presented as follows:

$$
\Pi_{2}^{\mathrm{SR}}(R)=m \Pi_{21}^{\mathrm{SR}}+n \Pi_{22}^{\mathrm{SR}}+(1-m-n) \int_{0}^{1} \Pi_{23}^{\mathrm{SR}} \mathrm{d} y .
$$

Furthermore, according to the relationship between $R$ and $D-Q$ (in the second stage, the market demand $D$ is deterministic), $\Pi_{2}^{\mathrm{SR}}(R)$ can be rewritten as follows:

$$
\Pi_{2}^{\mathrm{SR}}(R)= \begin{cases}\Pi_{2}^{\mathrm{SR} 1}(R), & R \geq D-Q>0, \\ \Pi_{2}^{\mathrm{SR} 2}(R), & R \leq D-Q,\end{cases}
$$

where

$$
\begin{aligned}
\Pi_{2}^{\mathrm{SR} 1}(R)= & m\left[p Q-c_{s}(D-Q)\right]+n\left[p D-c_{o}(Q+R-D)-\widetilde{w}_{2} R\right] \\
& +\int_{0}^{(D-Q) / R}\left[p(Q+y R)-c_{s}(D-Q-y R)-\widetilde{w}_{2} y R\right](1-m-n) \mathrm{d} y \\
& +\int_{(D-Q) / R}^{1}\left[p D-c_{o}(Q+y R-D)-\widetilde{w}_{2} y R\right](1-m-n) \mathrm{d} y, \\
\Pi_{2}^{\mathrm{SR} 2}(R)= & m\left[Q\left(p+c_{s}\right)-c_{s} D\right]+n\left[p(Q+R)-c_{s}(D-Q-R)-\widetilde{w}_{2} R\right] \\
& +\int_{0}^{1}\left[p(Q+y R)-c_{s}(D-Q-y R)-\widetilde{w}_{2} y R\right](1-m-n) \mathrm{d} y .
\end{aligned}
$$

In addition, since $\Pi_{2}^{\mathrm{SR} 1}(D-Q)=\Pi_{2}^{\mathrm{SR} 2}(D-Q), \Pi_{2}^{\mathrm{SR}}(R)$ is continuous in $R$. Combining cases of $D \leq Q$ and $D>Q$, the following lemma can be obtained via analyzing the properties of (5).

Lemma 2. Under the second stage of SRP and let $\Delta_{4}=$ $\sqrt{\left[\left(p+c_{s}+c_{o}\right)(1-m-n)\right] /\left[\left(\widetilde{w}_{2}+c_{o}\right)(1-m+n)\right]}$, the following hold true:
(1) When $\Delta_{4} \leq 1$, the retailer's optimal order quantity from supplier $2 R^{S R}=(D-Q)^{+}$

(2) When $\Delta_{4}>1$, the retailer's optimal order quantity from supplier $2 R^{S R}=\Delta_{4}(D-Q)^{+}$

Proof. The details can be found at Appendix D. From Lemma 2 , when $D>Q$, the retailer's emergency procurement quantity 
from unreliable supplier 2 would be definitely not less than $D$ $Q$ considering the supply disruption. And under SRP, two scenarios can be identified concerning the retailer's order quantity from the unreliable supplier 2 . That is, (i) when the emergency (shortage) cost is relatively higher (lower) or the supplier 2 is more likely to supply normally (i.e., higher values of $n$ ), $\Delta_{4}$ is more likely to be less than 1 (denoted as minor disruption scenario), implying that the retailer's emergency order quantity from the unreliable supplier 2 remains at $(D-Q)^{+}$. (ii) On the contrary, when supplier 2 is more possible to be entirely or partially disrupted, $\Delta_{4}$ would increase to exceed 1 (denoted as major disruption scenario), indicating that the retailer will set the order quantity from the supplier 2 equal to $\Delta_{4}(D-Q)^{+}$, aiming to avoid the shortage and minimize the operating cost.

5.2. Optimal Procurement Policy at the First Stage. In the first stage, the market demand $D$ is stochastic, and the retailer should order $Q$ units from the reliable supplier 1. Considering the impact of $\Delta_{4}$ on the retailer's order quantity from unreliable supplier 2 (from Lemma 2), the following two cases are considered:

(1) When $\Delta_{4} \leq 1$, then $R^{\mathrm{SR}}=(D-Q)^{+}$. Based on the relationship between $D$ and $Q$ mentioned in Lemma 2, we have the following two cases:

(i) When $D \leq Q$, we have $R^{\mathrm{SR}}=0$ and the retailer's profit equals $\Pi_{11}^{\mathrm{SR}}(Q)=p D-c_{o}(Q-D)-w_{1} Q$.

(ii) When $D>Q$, we have $R^{S R}=D-Q$, indicating that the retailer's profit equals $\Pi_{12}^{\mathrm{SR}}(Q)=m\left[Q\left(p+c_{s}\right)-c_{s} D\right]+n\left[D\left(p-\widetilde{w}_{2}\right)+\right.$ $\left.\widetilde{w}_{2} Q\right]+\int_{0}^{1}\left\{Q\left[\left(p+c_{s}\right)(1-y)+\widetilde{w}_{2} y\right]+[(p-\widetilde{w}\right.$ 2) $\left.\left.y-c_{s}(1-y)\right] D\right\}(1-m-n) \mathrm{d} y-w_{1} \mathrm{Q}$.

(2) When $\Delta_{4}>1$, then $R^{\mathrm{SR}}=\Delta_{4}(D-Q)^{+}$. Similarly,

(i) When $D \leq Q$, we have $R^{\mathrm{SR}}=0$ and $\Pi_{11}^{\mathrm{SR}}(Q)=p D-c_{o}(Q-D)-w_{1} Q$

(ii) When $D>Q$, we have $R^{S R}=\Delta_{4}(D-Q)$, indicating that the retailer's profit equals $\Pi_{12}^{\mathrm{SR}}(Q)=$ $m\left[Q\left(p+c_{s}\right)-c_{s} D\right]+n\left\{p D-\left[c_{o}\left(\Delta_{4}-1\right)+w_{2}\right.\right.$ $\left.\left.\Delta_{4}\right](D-Q)\right\}+\int_{0}^{1 / \Delta_{4}}\left\{p Q-\left[c_{s}\left(1-y \Delta_{4}\right)+w_{2}\right.\right.$ $\left.\left.y \Delta_{4}\right] \quad(D-Q)\right\}(1-m-n) \mathrm{d} y+\int_{1 / \Delta_{4}}^{1}\left\{p D-\left[c_{o}\right.\right.$ $\left.\left.\left(y \Delta_{4}-1\right)+w_{2} y \Delta_{4}\right](D-Q)\right\}(1-m-n) \mathrm{d} y-w_{1}$ Q.

Under the circumstance, the market demand $D$ is stochastic, indicating that the retailer's profit function including both $\Delta_{4} \leq 1$ and $\Delta_{4}>1$ can be presented by the following maximization problem:

$$
\Pi_{1}^{\mathrm{SR}}(Q)=\int_{0}^{Q} \Pi_{11}^{\mathrm{SR}}(Q) f(x) \mathrm{d} x+\int_{Q}^{+\infty} \Pi_{12}^{\mathrm{SR}}(Q) f(x) \mathrm{d} x .
$$

Thus, under different conditions of $\Delta_{4}$, the following theorem can be obtained.

Theorem 3. Under the first stage of SRP, let $\Delta_{5}=\left(p+c_{s}\right)$ $(1-n+m)+\widetilde{w}_{2}(1+n-m)$ and $\Delta_{6}=\left(p+c_{s}+c_{o}\right) m+$ $\sqrt{\left(p+c_{s}+c_{o}\right)(1-m-n)\left(\widetilde{w}_{2}+c_{o}\right)(1-m+n)}$; the following hold true:

(1) When $\Delta_{4} \leq 1$, then $Q^{S R}=F^{-1}\left[1-2\left(w_{1}+c_{o}\right) /\left(\Delta_{5}+\right.\right.$ $\left.\left.2 c_{o}\right)\right]$

(2) When $\Delta_{4}>1$, then $Q^{S R}=F^{-1}\left[1-\left(w_{1}+c_{o}\right) / \Delta_{6}\right]$

Proof. The details can be found at Appendix E. From Theorem 3, under both scenarios including minor and major disruption (corresponding to $\Delta_{4} \leq 1$ and $\Delta_{4}>1$, resp.), it is apparent that $Q^{S R}$ is negatively associated with $w_{1}$, while it is positively related to $p, c_{s}$, and $\widetilde{w}_{2}$. That is, the retailer would procure more quantities from the reliable supplier 1 to improve supply reliability if shortage cost plays a more important role or the selling price can be set higher values. Further, the comparison concerning $Q^{S R}$ under different scenarios can be presented in the following.

Corollary 2. In SRP, the retailer's order quantity from the reliable supplier 1 under minor disruption scenario (i.e., $\left.\Delta_{4} \leq 1\right)$ is not less than that under major disruption scenario (i.e., $\Delta_{4}>1$ ).

Proof. The details can be found at Appendix F. From Corollary 2, it is apparent that, under minor disruption scenario, the retailer would increase order quantity from the reliable supplier 1 compared with that of major disruption scenario. And combined with Lemma 2, it can be found that, under SRP, the retailer could assume different procurement policies according to the possibility of supply disruption. That is, (i) under minor disruption scenario, the retailer would assume a higher-order quantity from supplier 1 when demand is uncertain and accurately place order quantity from the unreliable supplier 2 based on $(D-Q)^{+}$after demand is realized. (ii) On the contrary, under major disruption scenario, the retailer would decrease the order quantity from supplier 1 (compared with that under minor disruption scenario) and set the order quantity from the supplier 2 equal to $\Delta_{4}(D-Q)^{+}$.

\section{Retailer's Optimal Procurement Policy under SS}

Under SS, the retailer simultaneously procures products from two suppliers when market demand is stochastic, aiming to take the advantage of lower wholesale prices (see Figure $1(\mathrm{~d})$ ). And considering supply disruption of supplier 2, the retailer's expected profit can be presented as follows:

(1) When the supplier 2 supply process is entirely disrupted (i.e., $y R=0$ ), the retailer could only apply $Q$ units offered by supplier 1 to satisfy the market demand. And we have $\Pi_{11}^{\mathrm{SS}}(Q, R)=E$ $\left[p \min (D, Q)-c_{s}(D-Q)^{+}-c_{o}(Q-D)^{+}-w_{1} Q\right]$.

(2) When the supplier's supply process is entirely normal (i.e., $y R=R$ ), the market demand can be met by the retailer's total received products $Q+R$ units. And we have $\Pi_{12}^{S S}(Q, R)=E\left[p \min (D, Q+R)-c_{s}\right.$ $\left.(D-Q-R)^{+}-c_{o}(Q+R-D)^{+}-w_{1} Q-w_{2} R\right]$. 
(3) When the supplier's supply process is partially disrupted; (i.e., $R>y R>0$ ), the retailer's total available products are $Q+y R$ units. And we have $\Pi_{13}^{S S}(Q, R)=$ $E\left[p \min (D, Q+y R)-c_{s}(D-Q-y R)^{+}-c_{o}(Q+y\right.$ $\left.R-D)^{+}-w_{1} Q-w_{2} y R\right]$.

Thus, the retailer's expected profit function under SS can be depicted as follows:

$$
\Pi_{1}^{\mathrm{SS}}(\mathrm{Q}, R)=m \Pi_{11}^{\mathrm{SS}}+n \Pi_{12}^{\mathrm{SS}}+(1-m-n) \int_{0}^{1} \Pi_{13}^{\mathrm{SS}} \mathrm{d} y .
$$

By analyzing the property of (9), the following theorem can be obtained.

Theorem 4. Under SS, when $n>m(1-m-n)$ and $\left(p+c_{s}-w_{1}\right) /\left(p+c_{s}-w_{2}\right)>(1-m+n) /(1-m-n), \quad$ the retailer's optimal order quantity from supplier 1 and supplier 2 can be obtained by the following expressions: $\int_{0}^{1} F\left(Q^{S S}+\right.$ $\left.y R^{S S}\right) d y+n F\left(Q^{S S}+R^{S S}\right)+m F\left(Q^{S S}\right)=\left(p+c_{s}-w_{1}\right) /(p+$ $\left.c_{s}+c_{o}\right)$ and $\int_{0}^{1} y F\left(Q^{S S}+y R^{S S}\right) d y+n F\left(Q^{S S}+R^{S S}\right) /(1-m-$ $n)=\left[(1-m+n)\left(p+c_{s}-w_{2}\right)\right] /\left[2\left(p+c_{s}+c_{o}\right)(1-m-n)\right]$.

Proof. The details can be found at Appendix G. From Theorem 4, under SS, it can be identified that both $Q^{S S}$ and $R^{\mathrm{SS}}$ are decreasing with the procurement prices of two supplies, including $w_{1}$ and $w_{2}$. Furthermore, according to Theorems $1-4$, it is possible to partially compare the retailer's order quantity from two suppliers under different sourcing strategies, which can be formulated as follows.

Corollary 3. When $n>m(1-m-n)$ and $\left(p+c_{s}-w_{1}\right) /(p+$ $\left.c_{s}-w_{2}\right)>(1-m+n) /(1-m-n)$, the following hold true.

(1) Regarding WPP-SR, if $w_{2} \geq\left[(1-m-n)\left(p+c_{s}\right)+w_{1}\right.$ $(1+m-n)] /[(1-n)(1+n-m)]$, then $R^{W S} \geq R^{S S}$

(2) With respect to WPP-DR, if $\widetilde{w}_{1} \geq p+c_{s}-c_{o}$, then $R^{S S} \leq R^{W D}$

(3) With respect to $S R P$ and $(m+n)^{2}-m+n>0$

(i) if $\Delta_{4} \leq 1$ and $\left(p+c_{s}-w_{2}\right) /\left(p+c_{s}+c_{o}\right) \leq 1-$ $2\left(w_{1}+c_{o}\right) /\left(\Delta_{5}+2 c_{0}\right)$, then $Q^{S S} \leq Q^{S R}$

(ii) if $\Delta_{4}>1$ and $\left(p+c_{s}-w_{2}\right) /\left(p+c_{s}+c_{o}\right) \leq$ $1-\left(w_{1}+c_{o}\right) / \Delta_{6}$, then $Q^{S S} \leq Q^{S R}$

Proof. The details can be found at Appendix H.

From Corollary 3, under mild assumptions, a series of results can be obtained to present differences of the retailer's procurement solutions under different sourcing strategies. Specifically, (1) with respect to WPP, provided that the selling price or $c_{s}$ is relatively lower, the retailer's order quantity from the unreliable supplier 2 under WPP (including WS and WD) is more than that of SS. In addition, if the wholesale price (emergency cost) of the reliable supplier 1 is relatively lower (higher), supplier 1 still plays a more important role under WPP than under SS. (2) Further, with the increase of the procurement (emergency cost) of the unreliable supplier 2, the retailer's order quantity from the reliable supplier 1 under SRP is likely to be greater than that under SS.

\section{Numerical Examples and Discussion}

Example 1. In this section, the following numerical examples are provided to emphasize the retailer's preferences towards backup sourcing and simultaneous sourcing strategies in an explicit manner. Specifically, referring to Giri and Bardhan [5] and Zeng and Xia [10], let the market demand $D$ follow a normal distribution with $\mu=150$ and $\sigma=5$. Other parameters are set as follows: $p=27, c_{s}=0.5, c_{o}=1, w_{1}=7, w_{2}=5, \widetilde{w}_{1}=20, \widetilde{w}_{2}=15$, $m=0.2$, and $n=0.4$. As a result, the form of $g(y)$ can be presented as follows:

$$
g(y)= \begin{cases}0.2, & y=0 \\ 0.4, & 0<y<1 \\ 0.4, & y=1\end{cases}
$$

In this numerical example, by changing the values of $p$, $c_{s}$, and $\mu$, the retailer's expected profit under different backup sourcing and simultaneous sourcing can be depicted in Figure 2 .

From Figure 2, under most of circumstances, the retailer achieves better performance under backup sourcing strategy than under simultaneous sourcing. It implies that when market demand is relatively stochastic, it is unwise for the retailer to simultaneously procure products from both suppliers. And more conclusions can be further obtained as follows:

(1) It is apparent that, under any sourcing strategy, the retailer can earn more expected profit when products can be sold at higher selling price $p$ (see Figure 2(a)). Further, when the selling price is relatively lower, the retailer could earn less profit margin, leading the retailer to adopt SRP for directly improving the supply reliability and avoiding expensive emergency replenishment from the reliable supplier. And as the selling price of products increases, the retailer is less sensitive to supply disruption and becomes more inclined to order quantities for achieving better performance. As a result, WPP-DR would be regarded as the optimal backup sourcing by the retailer for lower wholesale prices from the unreliable supplier.

(2) Along with the increase of unit shortage cost $c_{s}, \Pi_{1}^{\mathrm{SR}}$ and $\Pi_{1}^{\mathrm{WS}}$ and $\Pi_{1}^{\mathrm{SS}}$ decrease, while $\Pi_{1}^{\mathrm{WD}}$ remains the same (see Figure 2(b)). That is, since WPP-DR could ensure market demand to be entirely satisfied with expensive emergency replenishment from the reliable supplier, WPP-DR would be the priority strategy for the retailer when $c_{s}$ is relatively higher. Nevertheless, other sourcing strategies are still under the effects of shortage cost. Furthermore, when $c_{s}$ is insignificant, the retailer is better off under SRP to take the advantage of both supply reliability and lower wholesale prices of the reliable supplier. 


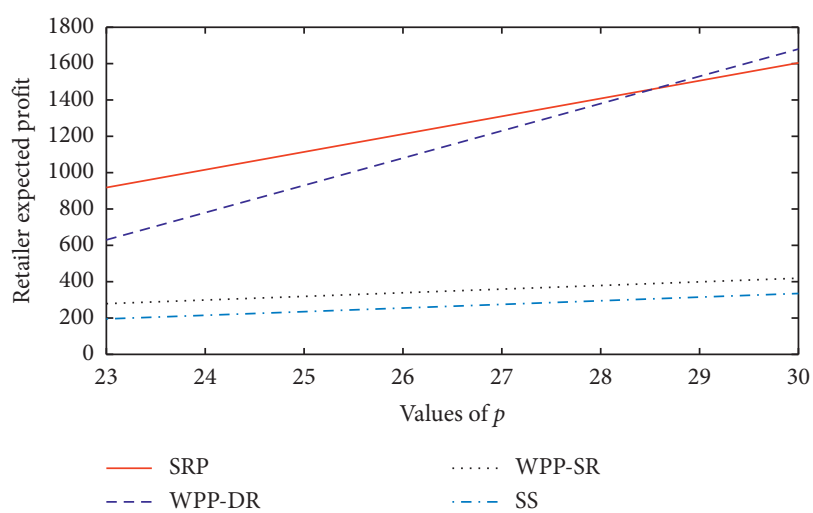

(a)

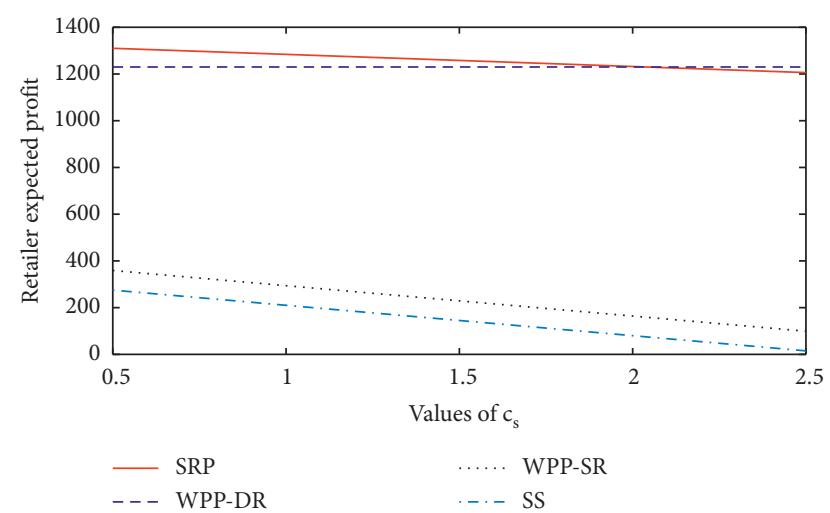

(b)

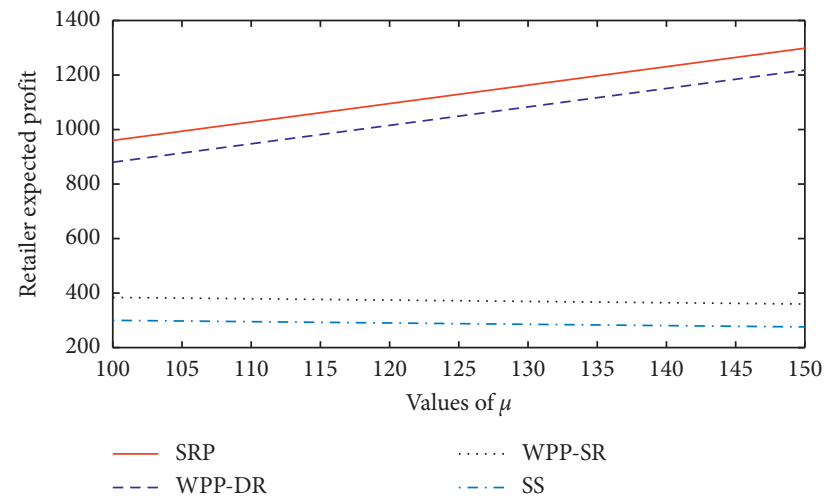

(c)

Figure 2: The retailer's expected profit under different values of $p, c_{s}$, and $\mu$.

(3) Higher values of $\mu$ lead to the increases of $\Pi_{1}^{S R}$ and $\Pi_{1}^{\mathrm{WD}}$, while resulting in the slight decreases of $\Pi_{1}^{\mathrm{SR}}$ and $\Pi_{1}^{\text {SS }}$ (see Figure $2(\mathrm{c})$ ). In other words, under SRP and WPP-DR, since one of the suppliers can be activated after the realization of market demand, the retailer could gain more profit by increasing the emergence quantity from the backup sourcing. On the contrary, the retailer under WPP-SR and SS would pay off more shortage costs in the same situation.

Example 2. In this example, via changing values of $m$ and $n$, this part aims to further verify the effects of supply disruption on the retailer's preferences towards different sourcing strategies. And two types of data concerning wholesale prices are further proposed including (i) $w_{1} \leq \widetilde{w}_{2}$ (i.e., $w_{1}=11, \widetilde{w}_{2}=16$ ) and (ii) $w_{1}>\widetilde{w}_{2}$ (i.e., $w_{1}=16$, $\left.\widetilde{w}_{2}=11\right)$, while other parameters remain the same in Example 1. Additionally, numerical results for $w_{1} \leq \widetilde{w}_{2}$ are presented in Figures 3(a) and 3(c), while others for $w_{1}>\widetilde{w}_{2}$ are shown in Figures 3(b) and 3(d).

According to Figure 3, it can be found that the retailer's expected profit under backup sourcing strategies can be improved to some degree when the supply process of the unreliable supplier 2 grows to be more reliable, i.e., the decrease of $m$ or the increase of $n$. Further, the retailer could earn more profit under SS when either $m$ or $n$ increase, indicating that the retailer can always benefit as long as the possibility of partial disruption reduces. In addition, in this example, the retailer is better off under backup sourcing than simultaneous sourcing. And the variation of $m$ and $n$ would impact the retailer's preference towards different backup sourcing strategies:

(1) From Figures 3(a) and 3(b), the retailer is under the combined effects of supply disruption (i.e., $m$ ) and the relationship of wholesale prices (i.e., between $w_{1}$ and $\left.\widetilde{w}_{2}\right)$. Specifically, if the unreliable supplier 2 is less likely to entirely disrupt (i.e., $m$ is relatively lower), the retailer would pay off more emergency cost to purchase products from the unreliable supplier 2, leading SRP to be the priority strategy. And along with the increase of $m$, the retailer is less convinced to receive products from the unreliable supplier 2 when demand is realized. In this case, the retailer would choose WPP-DR to regard the reliable supplier 1 as the backup supplier.

(2) From Figures 3(c) and 3(d), when the unreliable supplier 2 is less likely to work normally (i.e., $n$ is relatively lower), the retailer is better off under WPPDR. That is, the retailer would regard the unreliable supplier 2 as major sourcing for purchasing products with the lowest wholesale price and take the reliable supplier 2 as backup sourcing for ensuring all market 


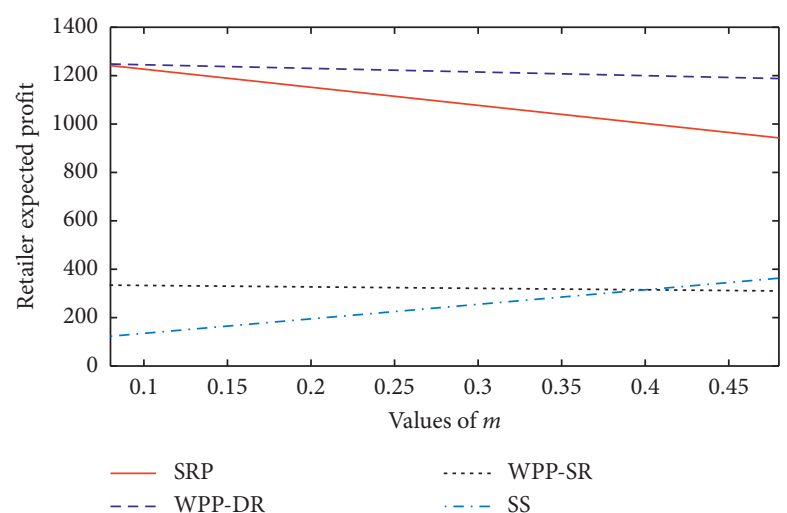

(a)

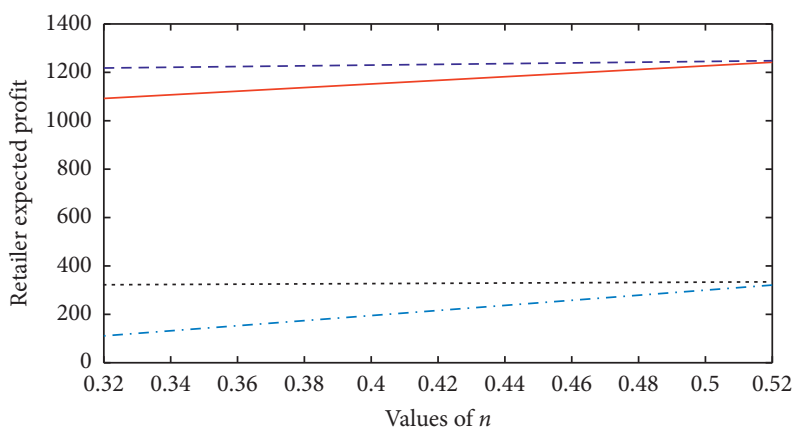

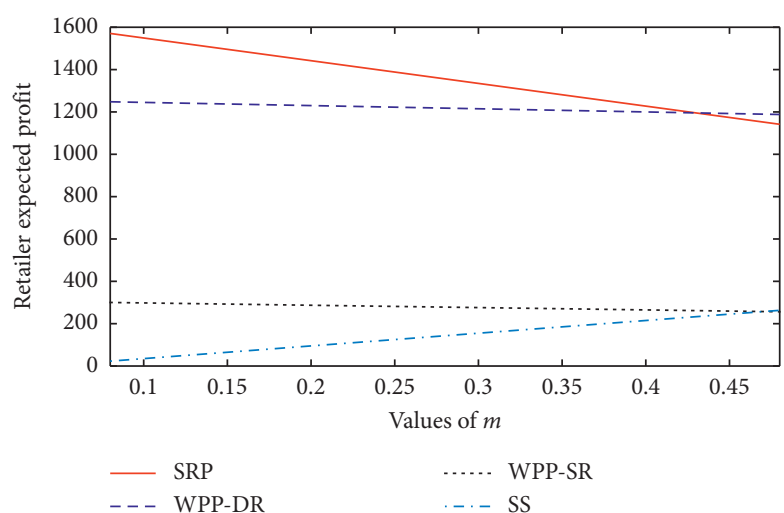

(b)

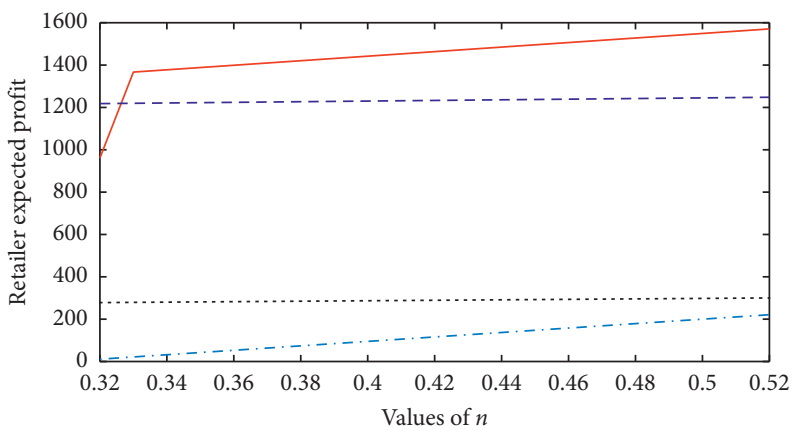

…. WPP-SR

(c)

FIGURE 3: The retailer's expected profit under different values of $m$ and $n$.

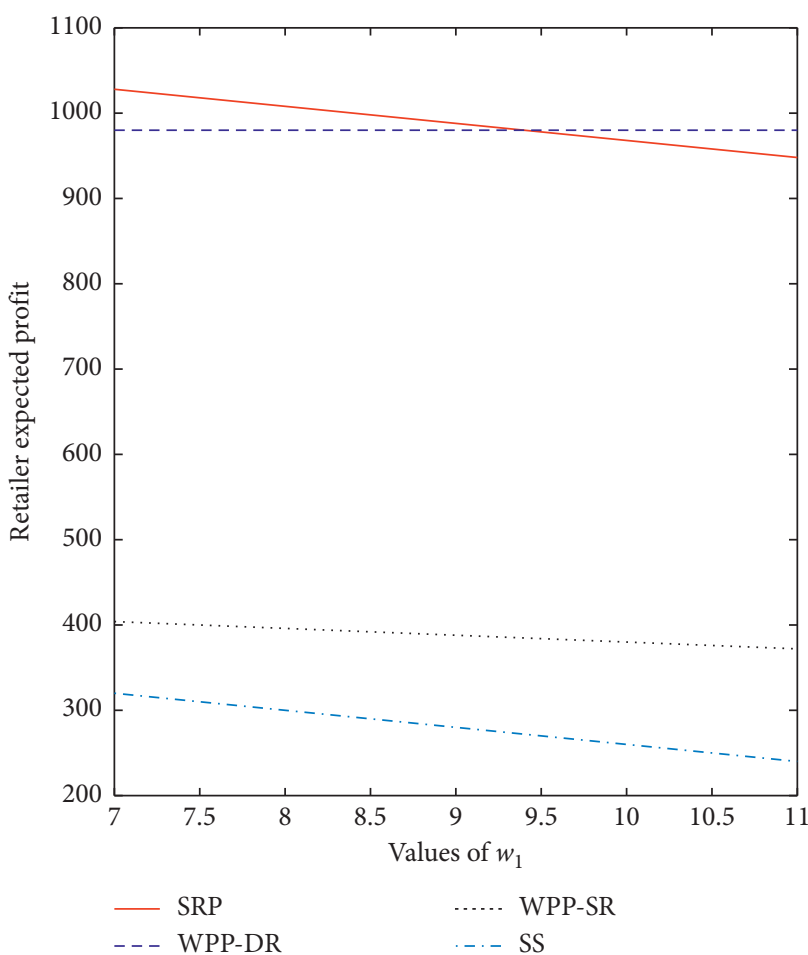

(a)

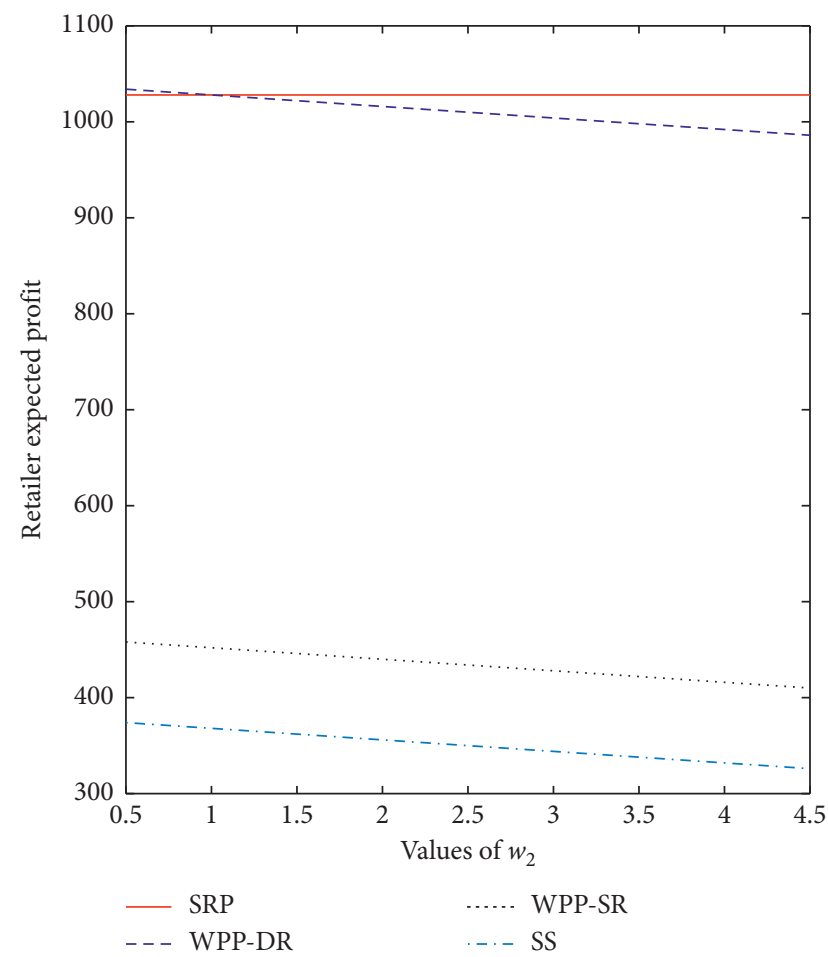

(b)

FIgURE 4: The retailer's expected profit under different values of $w_{1}$ and $w_{2}$. 
demand to be satisfied. Further, when the unreliable supplier 2 becomes more reliable, the retailer would prefer SPR, which regards supplier 1 as backup sourcing due to the lower wholesale price and its acceptable supply disruption. And this finding is irrelevant to the relationships between $w_{1}$ and $\widetilde{w}_{2}$.

Example 3. In this example, we aim to change values of wholesale prices to demonstrate the differences among different backup sourcing strategy and simultaneous sourcing policy. Apparently, along with the decreases of $\widetilde{w}_{1}$ and $\widetilde{w}_{2}$, all of three backup sourcing strategies would achieve better performance, while simultaneous sourcing strategy remains the same. Thus, in this example, we aim to change values of $w_{1}$ and $w_{2}$ to quantify their impacts. For simplicity, let $p=28, \mu=100$, and other parameters keep the same as those in Example 1.

From Figure 4, along with the increases of $w_{1}$ and $w_{2}$, the retailer's expected profit under both backup sourcing and simultaneous sourcing would be lowered. And since WPPDP and SRP are irrelevant to $w_{1}$ and $w_{2}$, respectively, it can be found that the retailer is better off under WPP-DP or SRP when $w_{1}$ or $w_{2}$ is relatively higher. In other words, compared with simultaneous sourcing strategy, the retailer is able to choose the specific backup sourcing strategies on the basis of wholesale price and the possibility of supply disruption. Additionally, unless the emergency prices are extremely higher, backup sourcing could perform better than simultaneous sourcing strategy.

\section{Conclusions}

Subject to the combined negative effects of supply disruption and stochastic demand, the retailer would suffer from supply shortage and loss of goodwill, which may significantly lower operational performances. To mitigate negative effects of supply uncertainty, this paper proposes different backup sourcing and simultaneous strategies, where backup sourcing includes wholesale price priority (WPP) and supply reliability priority (SRP). Although these different types of sourcing strategies have found their wide applications to prevent the retailer from stock risk, it is urgent for the retailer to identify the most appropriate sourcing strategy via trading off supply disruption and stochastic demand as well as wholesale prices:

(1) Under supply disruption and stochastic demand, two types of WPP strategies including WPP-SR and WPP-DR are available for the retailer to regard the unreliable supplier with lower wholesale price as the major sourcing. Specifically, (i) under WPP-SR, the reliable supplier is initiated after the occurrence of supply disruption for ensuring the retailer's order quantity from unreliable supplier to be always met. (ii) Under WPP-DR, the retailer would replenish from the reliable supplier after the realization of both supply and demand uncertainties, which guarantees the market demand to be entirely satisfied with more expensive emergency price. Besides, it is apparent that, along with the increases of selling price and shortage cost, the retailer definitely orders more quantity from the reliable supplier under WPP-SR, while it remains the same under WPP-DR. In addition, when the procurement or emergency price of the reliable supplier is relatively higher, the retailer's order quantity from the reliable supplier under WPP-SR is less than that of WPP-DR.

(2) Further, under SRP, there are two scenarios, including minor disruption and major disruption, when addressing the retailer's optimal order quantity from two suppliers. That is, (i) under minor disruption scenario, the unreliable supplier 2 is more likely to supply normally or offer a higher value of emergency price. Thus, the retailer would assume a higher-order quantity from supplier 1 when demand is uncertain and accurately place order quantity from the unreliable supplier 2 based on $(D-Q)^{+}$after demand is realized. (ii) Under major disruption scenario, the unreliable supplier 2 is more possible to be entirely or partially disrupted; the retailer would decrease the order quantity from supplier 1 (compared with that under minor disruption scenario) and set the order quantity from the supplier 2 equal to $\Delta_{4}(D-Q)^{+}$. In addition, under both minor and major disruption scenarios, the retailer would procure more quantities from the reliable supplier to improve supply reliability if shortage cost or selling price plays a more important role.

(3) Unlike existing studies under supply disruption, this paper systematically compares the retailer's preferences towards backup sourcing and simultaneous sourcing strategies via theoretical results and numerical examples. (i) Since WPP-DR could ensure market demand to be entirely satisfied by expensive emergency replenishment from supplier 1, the retailer would regard it as the optimal backup sourcing when selling price or shortage cost is relatively higher. On the contrary, the retailer would adopt SRP for directly improving the supply reliability and avoiding expensive emergency replenishment from the reliable supplier. (ii) When the unreliable supplier 2 is less likely to work normally, the retailer would regard WPP-DR the reliable supplier 2 as backup sourcing for ensuring all market demand to be satisfied. Otherwise, the retailer would prefer SPR, which regards supplier 2 as backup sourcing due to the lower wholesale price and its acceptable supply disruption. (iii) In addition, unless the emergency prices are extremely higher, backup sourcing could perform better than simultaneous sourcing.

\section{Appendix}

\section{A. The proof of Theorem 1}

By taking the first- and second-order derivative of $\Pi_{1}^{\mathrm{WS}}(R)$ with respect to $R$, we have $\mathrm{d} \Pi_{1}^{\mathrm{WS}}(R) / \mathrm{d} R=\bar{F}(R)-\Delta_{1}$ and 
$\mathrm{d}^{2} \Pi_{1}^{\mathrm{WS}}(R) / \mathrm{d} R^{2}=-f(R)\left(p+c_{s}+c_{o}\right)$, where $\Delta_{1}=[(1+m$ $\left.-n) w_{1}+(1+n-m) w_{2}+2 c_{o}\right] /\left[2\left(p+c_{s}+c_{o}\right)\right]$. Since $\mathrm{d}^{2} \Pi_{1}^{\mathrm{WS}}(R) / \mathrm{d} R^{2}<0, \Pi_{1}^{\mathrm{WS}}(R)$ is concave in $R$. Thus, by letting $\mathrm{d} \Pi_{1}^{\mathrm{WS}}(R) / \mathrm{d} R=0$, the retailer's optimal order quantity from the unreliable supplier $2 \bar{F}\left(R^{\mathrm{WS}}\right)=\Delta_{1}$.

\section{B. The proof of Theorem 2}

By taking the first- and second-order derivative of $\Pi_{1}^{\mathrm{WD}}(R)$ with respect to $R$, we have $\mathrm{d} \Pi_{1}^{\mathrm{WD}}(R) / \mathrm{d} R=n \bar{F}(R)+(1-n-$ m) $\int_{0}^{1} y \bar{F}(y R) \mathrm{d} y-(1+n-m)\left(c_{o}+w_{2}\right) / 2\left[\left(c_{o}+\widetilde{w}_{1}\right)\right]$ and $\mathrm{d}^{2}$ $\Pi_{1}^{\mathrm{WD}}(R) / \mathrm{d} R^{2}=-\left(c_{o}+\widetilde{w}_{1}\right) \quad\left[n f(R)+(1-n-m) \int_{0}^{1} y^{2} f(y R)\right.$ $\mathrm{d} y$ ]. Since $\mathrm{d}^{2} \Pi_{1}^{\mathrm{WD}}(R) / \mathrm{d} R^{2}<0, \Pi_{1}^{\mathrm{WD}}(R)$ is concave in $R$. Thus, by letting $\mathrm{d} \Pi_{1}^{\mathrm{WD}}(R) / \mathrm{d} R=0$, the retailer's optimal order quantity $R^{\mathrm{WD}}$ from the unreliable supplier $2 n \bar{F}$ $\left(R^{\mathrm{WD}}\right)+(1-n-m) \int_{0}^{1} y \bar{F}\left(y R^{\mathrm{WD}}\right) \mathrm{d} y=(1+n-m)\left(c_{o}+w_{2}\right) /$ $\left[2\left(c_{o}+\widetilde{w}_{1}\right)\right]$.

\section{The proof of Corollary 1}

According to the assumption, it is apparent that $\bar{F}(D)$ is decreasing with $D$. And based on Theorem 2, two cases can be discussed as follows:

(1) For $y \in(0,1)$, we have $\bar{F}\left(y R^{\mathrm{WD}}\right)>\bar{F}\left(R^{\mathrm{WD}}\right)$, indicating that $\int_{0}^{1} y \bar{F}\left(y R^{\mathrm{WD}}\right) \mathrm{d} y>\bar{F}\left(R^{\mathrm{WD}}\right) \int_{0}^{1} y \mathrm{~d} y=\bar{F}$ $\left(R^{\mathrm{WD}}\right) / 2$. Thus, it can be inferred that $(1+n-m)$ $\left(c_{o}+w_{2}\right) /\left[2\left(c_{o}+\widetilde{w}_{1}\right)\right]=n \bar{F}\left(R^{\mathrm{WD}}\right)+(1-n-m) \int_{0}^{1}$ $y \bar{F}\left(y R^{\mathrm{WD}}\right) \mathrm{d} y>(1+n-m) \bar{F}\left(R^{\mathrm{WD}}\right) / 2$, resulting in $\Delta_{2}>\bar{F}\left(R^{\mathrm{WD}}\right)$, where $\Delta_{2}=\left(c_{o}+w_{2}\right) /\left(c_{o}+\widetilde{w}_{1}\right)$.

(2) For $y \in(0,1)$, we have $\bar{F}\left(y R^{\mathrm{WD}}\right)<1$, implying that $\int_{0}^{1} y \bar{F}\left(y R^{\mathrm{WD}}\right) \mathrm{d} y<\int_{0}^{1} y \mathrm{~d} y=0.5$. Thus, it can be inferred that $(1+n-m)\left(c_{o}+w_{2}\right) / 2\left(c_{o}+\widetilde{w}_{1}\right)=n \bar{F}$ $\left(R^{\mathrm{WD}}\right)+(1-n-m) \int_{0}^{1} y \bar{F}\left(y R^{\mathrm{WD}}\right) \mathrm{d} y<n \bar{F}\left(R^{\mathrm{WD}}\right)$ $+(1-n-m) / 2$, resulting in $\bar{F}\left(R^{\mathrm{WD}}\right)>\Delta_{3}$, where $\Delta_{3}=\left[(1+n-m) w_{2}-(1-n-m) \widetilde{w}_{1}+2 n c_{o}\right] /[2 n$ $\left.\left(c_{o}+\widetilde{w}_{1}\right)\right]$.

In addition, according to the expression of $\bar{F}\left(R^{\mathrm{WS}}\right)=\Delta_{1}$ in Theorem 1, the following results can be derived:

(1) If $\Delta_{1} \geq \Delta_{2}$, then $\bar{F}\left(R^{\mathrm{WS}}\right)=\Delta_{1} \geq \Delta_{2}>\bar{F}\left(R^{\mathrm{WD}}\right)$ and $R^{\mathrm{WS}} \leq R^{\mathrm{WD}}$

(2) If $\Delta_{1}<\Delta_{3}$, then $\bar{F}\left(R^{\mathrm{WS}}\right)=\Delta_{1} \leq \Delta_{3}<\bar{F}\left(R^{\mathrm{WD}}\right)$ and $R^{\mathrm{WS}} \geq R^{\mathrm{WD}}$

\section{The proof of Lemma 2}

When $D>Q$, two situations can be classified based on the relationship between $D-Q$ and $R$.

(1) We have $\mathrm{d}^{2} \Pi_{2}^{\mathrm{SR} 1}(R) / \mathrm{d} R^{2}=-(D-Q)^{2}\left(p+c_{s}+c_{o}\right)$ $(1-m-n) / R^{3}<0$. Via letting $\mathrm{d} \Pi_{2}^{\text {SR1 }}(R) / \mathrm{d} R=0$, we have $\quad R^{\mathrm{SR} 1}=\Delta_{4}(D-Q)$, where $\Delta_{4}=$ $\sqrt{\left[\left(p+c_{s}+c_{o}\right)(1-m-n)\right] /\left[\left(\widetilde{w}_{2}+c_{o}\right)(1-m+n)\right]}$. Under the circumstance, considering that $R \geq D-Q$, two cases can be obtained when comparing $\Delta_{4}$ with 1. That is, if $\Delta_{4} \leq 1$, then $R^{S R 1}=D-Q$; otherwise, $R^{\mathrm{SR} 1}=\Delta_{4}(D-Q)$.
(2) Furthermore, we have $\mathrm{d} \Pi_{2}^{\mathrm{SR} 2}(R) / \mathrm{d} R=$ $\left(p+c_{s}-\widetilde{w}_{2}\right)(1-m+n) / 2>0$. That is, $\Pi_{2}^{\mathrm{SR} 2}(R)$ is increasing in $R$, implying that $R^{\mathrm{SR} 2}=D-Q$.

Thus, under the circumstance of $D>Q$, the following results can be obtained:

(1) When $\Delta_{4} \leq 1$, both $\Pi_{2}^{\mathrm{SR} 1}(R)$ and $\Pi_{2}^{\mathrm{SR} 2}(R)$ can be maximized at $D-Q$, implying that the retailer's optimal order quantity for supplier $2 R^{\mathrm{SR}}=D-Q$

(2) When $\Delta_{4}>1$, since $\Pi_{2}^{\mathrm{SR} 1}\left(\Delta_{4}(D-Q)\right) \geq \Pi_{2}^{\mathrm{SR} 1}(D-$ $Q)=\Pi_{2}^{\mathrm{SR} 2}(D-Q)$, the retailer's optimal order quantity for supplier $2 R^{\mathrm{SR}}=\Delta_{4}(D-Q)$

Furthermore, considering $R^{S R}=0$ when $D \leq Q$, the following result can be formulated:

(1) When $\Delta_{4} \leq 1$, the retailer's optimal order quantity for supplier $2 R^{\mathrm{SR}}=(D-Q)^{+}$

(2) When $\Delta_{4}>1$, the retailer's optimal order quantity for supplier $2 R^{\mathrm{SR}}=\Delta_{4}(D-Q)^{+}$

\section{E. The proof of Theorem 3}

According to the value of $\Delta_{4}$, the following two cases can be obtained:

(1) When $\Delta_{4} \leq 1$, we have $\mathrm{d}^{2} \Pi_{1}^{\mathrm{SR}}(Q) / \mathrm{dQ}^{2}=-f$

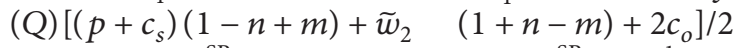
$<0$. Let $\mathrm{d} \Pi_{1}^{\mathrm{SR}}(\mathrm{Q}) / \mathrm{dQ}=0$; then $Q^{\mathrm{SR}}=F^{-1}[1-$ $\left.2\left(w_{1}+c_{o}\right) /\left(\Delta_{5}+2 c_{o}\right)\right]$, where $\Delta_{5}=\left(p+c_{s}\right)(1-n+$ $m)+\widetilde{w}_{2}(1+n-m)$.

(2) When $\Delta_{4}>1$, we have $\mathrm{d}^{2} \Pi_{1}^{\mathrm{SR}}(Q) / \mathrm{dQ}^{2}=-0.5 f(Q)$ $\left[\left(\widetilde{w}_{2}+c_{o}\right)(1+n-m) \Delta_{4}^{2}+\left(p+c_{s}+c_{o}\right)\left(2 \Delta_{4} m-m-\right.\right.$ $n+1)] / \Delta_{4}<0$. Let $\mathrm{d} \Pi_{1}^{\mathrm{SR}}(\mathrm{Q}) / \mathrm{dQ}=0$; then $Q^{\mathrm{SR}}=$ $F^{-1}\left[1-\left(w_{1}+c_{o}\right) / \Delta_{6}\right]$, where $\Delta_{6}=\left(p+c_{s}+c_{o}\right) m+$ $\sqrt{\left(p+c_{s}+c_{o}\right)(1-m-n)\left(\widetilde{w}_{2}+c_{o}\right)(1-m+n)}$.

\section{F. The proof of Corollary 2}

According to values of $Q^{\mathrm{SR}}$ in Theorem 3, let $g_{1}=1-2\left(w_{1}+\right.$ $\left.c_{o}\right) /\left(\Delta_{5}+2 c_{o}\right)-\left[1-\left(w_{1}+c_{o}\right) / \Delta_{6}\right]=\left(w_{1}+c_{o}\right)\left(\Delta_{5}+2 c_{o}-\right.$ $\left.2 \Delta_{6}\right) /\left[\Delta_{6}\left(\Delta_{5}+2 c_{o}\right)\right]$. Since $\Delta_{5}>0$ and $\Delta_{6}>0$, we should only analyze the sign of $\Delta_{5}+2 c_{o}-2 \Delta_{6}$. Based on values of $\Delta_{5}$ and $\Delta_{6}$, we have $\Delta_{5}+2 c_{o}-2 \Delta_{6}=\left[\sqrt{\left(p+c_{s}+c_{o}\right)(1-m-}\right.$ $\left.n)-\sqrt{\left(\widetilde{w}_{2}+c_{o}\right)(1-m+n)}\right]^{2} \geq 0$, indicating that $g_{1}>0$ and $F^{-1}\left[1-2\left(w_{1}+c_{o}\right) /\left(\Delta_{5}+2 c_{o}\right)\right] \geq F^{-1}\left[1-\left(w_{1}+c_{o}\right) / \Delta_{6}\right]$. Thus, in SRP, the retailer's order quantity from the reliable supplier 1 under minor disruption scenario (i.e., $\Delta_{4} \leq 1$ ) is not less than that under major disruption scenario (i.e., $\left.\Delta_{4}>1\right)$.

\section{G. The proof of Theorem 4}

According to equation (9), we have $\partial^{2} \Pi_{1}^{S S}(Q, R) / \partial R^{2}=$ $-\left(p+c_{s}+c_{o}\right)\left[n f(Q+R)+(1-m-n) \int_{0}^{1} y^{2} f(Q+y R) \mathrm{d} y\right]$, $\partial^{2} \Pi_{1}^{S S}(Q, R) / \partial Q^{2}=-\left(p+c_{s}+c_{o}\right) \quad[m f(Q)+n f(Q+R)+$ $\left.(1-m-n) \int_{0}^{1} f(Q+y R) \mathrm{d} y\right]$, and $\partial^{2} \Pi_{1}^{S S}(Q, R) / \partial Q \partial R=$ $-\left(p+c_{s}+c_{o}\right) \quad\left[n f(Q+R)+(1-m-n) \quad \int_{0}^{1} y f(Q+y R)\right.$ $\mathrm{d} y$ ]. Thus, the Hessian matrix $=\left(\partial^{2} \Pi_{1}^{S S}(Q, R) / \partial\right.$ 
$\left.R^{2}\right)\left(\partial^{2} \Pi_{1}^{S S}(Q, R) / \partial Q^{2}\right)-\left(\partial^{2} \Pi_{1}^{S S}(Q, R) / \partial Q \partial R\right)^{2}>\left(p+c_{s}+\right.$ $\left.c_{o}\right)^{2}(1-m-n)\left[L_{11}+(1-m-n) L_{12}\right]$, where $L_{11}=n f(Q+$ R) $\int_{0}^{1}(1-y)^{2} f(Q+y R) \mathrm{d} y>0$ and $L_{12}=\int_{0}^{1} f(Q+y R)$ $\mathrm{d} y \int_{0}^{1} y^{2} f(Q+y R) \mathrm{d} y-\left[\int_{0}^{1} y f(Q+y R) \mathrm{d} y\right]^{2}=\left(1 / R^{2}\right)\{[F$ $(Q+R)-F(Q)] \quad\left[F(Q+R)-\int_{0}^{1} 2 y F(Q+y R) \mathrm{d} y\right]-[F$ $\left.\left.(Q+R)-\int_{0}^{1} \quad F(Q+y R) \mathrm{d} y\right]^{2}\right\}, \quad$ where $\quad F(Q)<\int_{0}^{1} F$ $(\mathrm{Q}+y R) \mathrm{d} y$.

Furthermore, by letting $\partial \Pi_{1}^{S S}(Q, R) / \partial Q$ and $\partial \Pi_{1}^{S S}(Q, R) / \partial R$ equal zero, respectively, we have $\int_{0}^{1} F(Q+y R) \mathrm{d} y=\left(p+c_{s}-w_{1}\right) /\left(p+c_{s}+c_{o}\right)-[n F(Q+$ $R)+m F(Q)]$ and $\int_{0}^{1} y F(Q+y R) \mathrm{d} y=(1-m+n)\left(p+c_{s}-\right.$ $\left.w_{2}\right) /\left[2\left(p+c_{s}+c_{o}\right)(1-m-n)\right]-n F \quad(Q+R) /(1-m-n)$. Thus, provided that both $n>m(1-m-n)$ and $(p+$ $\left.c_{s}-w_{1}\right) /\left(p+c_{s}-w_{2}\right)>(1-m+n) /(1-m-n)$, we have $\int_{0}^{1} 2 y F(Q+y R) \mathrm{d} y<\int_{0}^{1} F(Q+y R) \mathrm{d} y, L_{12}>0$, and $\left(\partial^{2} \Pi_{1}^{S S}\right.$ $\left.(Q, R) / \partial R^{2}\right)\left(\partial^{2} \Pi_{1}^{S S}(Q, R) / \partial Q^{2}\right)-\left(\partial^{2} \Pi_{1}^{S S}(Q, R) / \partial Q \partial R\right)^{2}>0$, implying that the optimal order quantity from supplier 1 and supplier 2 can be obtained via simultaneously letting $\partial \Pi_{1}^{\mathrm{SS}}(\mathrm{Q}, R) / \partial \mathrm{Q}$ and $\partial \Pi_{1}^{\mathrm{SS}}(\mathrm{Q}, R) / \partial R$ equal zero.

\section{H. The proof of Corollary 3}

Under the assumption of $n>m(1-m-n)$ and $\left(p+c_{s}-\right.$ $\left.w_{1}\right) /\left(p+c_{s}-w_{2}\right)>(1-m+n) /(1-m-n)$ derived from Theorem 4 , values of $Q^{\mathrm{SS}}$ and $R^{\mathrm{SS}}$ can be uniquely obtained, making it possible to compare the retailer's order quantity under different backup sourcing strategies:

(1) With respect to WPP-SR, we have $F\left(R^{\mathrm{WS}}\right)=1-\Delta_{1}$ from Theorem 1. And from Theorem $4, R^{\mathrm{SS}}$ is subject to both $\int_{0}^{1} F\left(Q^{\mathrm{SS}}+y R^{\mathrm{SS}}\right) \mathrm{d} y+n F\left(Q^{\mathrm{SS}}+R^{\mathrm{SS}}\right)+m F$ $\left(Q^{\mathrm{SS}}\right)=\left(p+c_{s}-w_{1}\right) /\left(p+c_{s}+c_{o}\right)$ and $\int_{0}^{1} y F\left(Q^{\mathrm{SS}}+\right.$ $\left.y R^{\mathrm{SS}}\right) \mathrm{d} y+n F\left(Q^{\mathrm{SS}}+R^{\mathrm{SS}}\right) /(1-m-n)=(1-m+n)$ $\left(p+c_{s}-w_{2}\right) /\left[2\left(p+c_{s}+c_{o}\right) \quad(1-m-n)\right]$, which can be transformed into $F\left(R^{S S}\right)<\left(p+c_{s}-\right.$ $\left.w_{1}\right) /\left[n\left(p+c_{s}+c_{o}\right)\right]$ and $F\left(R^{S S}\right)<(1-m+n)(p+$ $\left.c_{s}-w_{2}\right) /\left[2 n\left(p+c_{s}+c_{o}\right)\right], \quad$ respectively. Further, $\left(p+c_{s}-w_{1}\right) /\left[n\left(p+c_{s}+c_{o}\right)\right]-(1-m+n)\left(p+c_{s}-\right.$ $\left.w_{2}\right) /\left[2 n\left(p+c_{s}+c_{o}\right)\right]=\left(p+c_{s}-w_{2}\right) \quad\left[2\left(p+c_{s}-\right.\right.$ $\left.\left.w_{1}\right) /\left(p+c_{s}-w_{2}\right)-(1-m+n)\right] /\left[2 n\left(p+c_{s}+c_{o}\right)\right]$ $>\left(p+c_{s}-w_{2}\right) /[2(1-m+n) /(1-m-n)-(1-$ $m+n)] /\left[2 n\left(p+c_{s}+c_{o}\right)\right]>0$, which results in $F\left(R^{S S}\right)<\min \left\{\left(p+c_{s}-w_{1}\right) /\left[n\left(p+c_{s}+c_{o}\right)\right], \quad(1-m+\right.$ $\left.n)\left(p+c_{s}-w_{2}\right) /\left[2 n\left(p+c_{s}+c_{o}\right)\right]\right\}=(1-m+n)(p+$ $\left.c_{s}-w_{2}\right) /\left[2 n\left(p+c_{s}+c_{o}\right)\right]$. Thus, provided that 1$\Delta_{1} \geq(1-m+n)\left(p+c_{s}-w_{2}\right) /\left[2 n\left(p+c_{s}+c_{o}\right)\right]$ (i.e., $w_{2}$ $\geq\left[\begin{array}{ll}(1-m-n) & \left.\left(p+c_{s}\right)+w_{1}(1+m-n)\right] /[(1-n) \quad(1+\end{array}\right.$ $n-m)]$, we have $F\left(R^{\mathrm{WS}}\right) \geq F\left(R^{\mathrm{SS}}\right)$ and $R^{\mathrm{WS}} \geq R^{\mathrm{SS}}$.

(2) With respect to WPP-DR, from Theorem 2 , we have $n \bar{F}\left(R^{\mathrm{WD}}\right)+(1-n-m) \int_{0}^{1} y \bar{F}\left(y R^{\mathrm{WD}}\right) \mathrm{d} y=(1+n-$ $m)\left(c_{o}+w_{2}\right) /\left[2\left(c_{o}+\widetilde{w}_{1}\right)\right]$. And from Theorem 4 , it is apparent that $(1-m+n)\left(p+c_{s}-w_{2}\right) /\left[2\left(p+c_{s}+\right.\right.$ $\left.\left.c_{o}\right)(1-m-n)\right]=\int_{0}^{1} y F\left(Q^{S S}+y R^{S S}\right) \mathrm{d} y+n F\left(Q^{S S}+\right.$ $\left.R^{\mathrm{SS}}\right) /(1-m-n)>\int_{0}^{1} y F\left(y R^{\mathrm{SS}}\right) \mathrm{d} y+n F\left(R^{\mathrm{SS}}\right) /(1-$ $m-n)$, which yields $(1-m-n) \int_{0}^{1} y \bar{F}\left(y R^{S S}\right) \mathrm{d} y+$ $n \bar{F}\left(R^{S S}\right)>(1-m+n)\left(c_{o}+w_{2}\right) /\left[2\left(p+c_{s}+c_{o}\right)\right]$. As a result, provided that $(1-m+n)\left(c_{o}+w_{2}\right) /[2(p+$ $\left.\left.c_{s}+c_{o}\right)\right] \geq(1+n-m)\left(c_{o}+w_{2}\right) /\left[2\left(c_{o}+\widetilde{w}_{1}\right)\right]$ (i.e., $\left.\widetilde{w}_{1} \geq p+c_{s}\right)$, we have $R^{\mathrm{SS}} \leq R^{\mathrm{WD}}$.

(3) According to Theorem 4, it is apparent that $Q^{\mathrm{SS}}$ is subject to both $\left(p+c_{s}-w_{1}\right) /\left(p+c_{s}+c_{o}\right)>(1+m$ $+n) \int_{0}^{1} F\left(Q^{S S}\right) \mathrm{d} y$ and $(1-m+n)\left(p+c_{s}-w_{2}\right) /[2(p$ $\left.+c_{s}+c_{o}\right)(1-m-n)>F\left(Q^{\mathrm{SS}}\right) / 2+n F\left(Q^{\mathrm{SS}}\right) /(1-m$ $-n)$, which can be transformed into $F\left(Q^{\mathrm{SS}}\right)<(p+$ $\left.c_{s}-w_{1}\right) /\left[\left(p+c_{s}+c_{o}\right)(1+m+n)\right]$ and $F\left(Q^{S S}\right)<$ $\left(p+c_{s}-w_{2}\right) /\left(p+c_{s}+c_{o}\right)$, respectively. Further, $(p$ $\left.+c_{s}-w_{1}\right) /\left[\left(p+c_{s}+c_{o}\right)(1+m+n)\right]-\left(p+c_{s}-w_{2}\right) /(p$ $\left.\left.+c_{s}+c_{o}\right)=\left(p+c_{s}-w_{2}\right) /\left(p+c_{s}+c_{o}\right)(1+m+n)\right)$ $\left[\left(p+c_{s}-w_{1}\right) /\left(p+c_{s}-w_{2}\right)-(1+m+n)\right] /\left[\left(p+c_{s}+\right.\right.$ $\left.\left.c_{o}\right)(1+m+n)\right]>\left(p+c_{s}-w_{2}\right) /\left(p+c_{s}+c_{o}\right)(1+m+n)$ $[(1-m+n / 1-m-n)-(1+m+n)] /\left[\left(p+c_{s}+c_{o}\right)(1\right.$ $+m+n)]=\left(p+c_{s}-w_{2}\right)\left[(m+n)^{2}-m+n\right] /\left\{\left(p+c_{s}+\right.\right.$ $\left.\left.c_{o}\right)\left[1-(m+n)^{2}\right]\right\}$. It implies that when $(m+n)^{2}-$ $m+n>0$, we have $F\left(Q^{S S}\right)<\min \left\{\left(p+c_{s}-w_{1}\right) /\left(p+c_{s}\right.\right.$ $\left.\left.+c_{o}\right)(1+m+n),\left(p+c_{s}-w_{2}\right) /\left(p+c_{s}+c_{o}\right)\right\}=\left(p+c_{s}-\right.$ $\left.w_{2}\right) /\left(p+c_{s}+c_{o}\right)$. Further, from Theorem 3 , two cases can be obtained as follows:

(i) If $\Delta_{4} \leq 1$ and $\left(p+c_{s}-w_{2}\right) /\left(p+c_{s}+c_{o}\right)$ $\leq 1-2\left(w_{1}+c_{o}\right) /\left(\Delta_{5}+2 c_{o}\right)$, then $F\left(Q^{S S}\right)<$ $F\left(Q^{\mathrm{SR}}\right)$ and $Q^{\mathrm{SS}} \leq Q^{\mathrm{SR}}$.

(ii) If $\Delta_{4}>1$ and $\left(p+c_{s}-w_{2}\right) /\left(p+c_{s}+c_{o}\right) \leq$ $1-\left(w_{1}+c_{o}\right) / \Delta_{6}$, then $F\left(Q^{S S}\right)<F\left(Q^{S R}\right)$ and $Q^{\mathrm{SS}} \leq Q^{\mathrm{SR}}$.

\section{Data Availability}

All data in the manuscript are fully available without any restriction and all related data are within the paper.

\section{Conflicts of Interest}

The authors declare that there are no conflicts of interest.

\section{Acknowledgments}

The authors acknowledge the support from the National Natural Science Foundation of China under Grant nos. 71701049 and 71871159, the Middle-Aged and Young Teachers Education and Research Project Foundation of Fujian Province under Grant no. JAS19028, and the Major Project Funding for Social Science Research Base in Fujian Province Social Science Planning under Grant no. FJ2018JDZ024.

\section{References}

[1] J. Hou, A. Z. Zeng, and L. Sun, "Backup sourcing with capacity reservation under uncertain disruption risk and minimum order quantity," Computers \& Industrial Engineering, vol. 103, pp. 216-226, 2017. 
[2] T. Sawik, "Joint supplier selection and scheduling of customer orders under disruption risks: single vs. dual sourcing," Omega, vol. 43, pp. 83-95, 2014.

[3] X. Li, "Optimal procurement strategies from suppliers with random yield and all-or-nothing risks," Annals of Operations Research, vol. 257, no. 1-2, pp. 167-181, 2017.

[4] S. Zhang, P. Zhang, and M. Zhang, "Fuzzy emergency model and robust emergency strategy of supply chain system under random supply disruptions," Complexity, vol. 2019, Article ID 3092514, 10 pages, 2019.

[5] B. C. Giri and S. Bardhan, "Coordinating a supply chain under uncertain demand and random yield in presence of supply disruption," International Journal of Production Research, vol. 53, no. 16, pp. 5070-5084, 2015.

[6] L. Silbermayr and S. Minner, "Dual sourcing under disruption risk and cost improvement through learning," European Journal of Operational Research, vol. 250, no. 1, pp. 226-238, 2016.

[7] L. Ma, W. Xue, Y. Zhao, and Q. Zeng, "Loss-averse newsvendor problem with supply risk," Journal of the Operational Research Society, vol. 67, no. 2, pp. 214-228, 2016.

[8] X. Li and Y. Li, "On the loss-averse dual-sourcing problem under supply disruption," Computers \& Operations Research, vol. 100, pp. 301-313, 2018.

[9] M. Kumar, P. Basu, and B. Avittathur, "Pricing and sourcing strategies for competing retailers in supply chains under disruption risk," European Journal of Operational Research, vol. 265 , no. 2 , pp. $533-543,2018$.

[10] A. Z. Zeng and Y. Xia, "Building a mutually beneficial partnership to ensure backup supply," Omega, vol. 52, pp. 77-91, 2015.

[11] A. J. Schmitt, L. V. Snyder, and Z.-J. M. Shen, "Inventory systems with stochastic demand and supply: properties and approximations," European Journal of Operational Research, vol. 206, no. 2, pp. 313-328, 2010.

[12] S. Y. Tang, H. Gurnani, and D. Gupta, "Managing disruptions in decentralized supply chains with endogenous supply process reliability," Production and Operations Management, vol. 23, no. 7, pp. 1198-1211, 2014.

[13] P. Ray and M. Jenamani, "Sourcing decision under disruption risk with supply and demand uncertainty: a newsvendor approach," Annals of Operations Research, vol. 237, no. 1-2, pp. 237-262, 2014.

[14] K. Inderfurth and G. P. Kiesmüller, "Exact and heuristic linear-inflation policies for an inventory model with random yield and arbitrary lead times," European Journal of Operational Research, vol. 245, no. 1, pp. 109-120, 2015.

[15] Z. Yin and C. Wang, "Strategic cooperation with a backup supplier for the mitigation of supply disruptions," International Journal of Production Research, vol. 56, no. 12, pp. 4300-4312, 2018.

[16] J. Hou, A. Z. Zeng, and L. Zhao, "Coordination with a backup supplier through buy-back contract under supply disruption," Transportation Research Part E: Logistics and Transportation Review, vol. 46, no. 6, pp. 881-895, 2010.

[17] L. Qi, “A continuous-review inventory model with random disruptions at the primary supplier," European Journal of Operational Research, vol. 225, no. 1, pp. 59-74, 2013.

[18] H. Yu, A. Z. Zeng, and L. Zhao, "Single or dual sourcing: decision-making in the presence of supply chain disruption risks," Omega, vol. 37, no. 4, pp. 788-800, 2009.

[19] Y. Zhang and X. Wang, "Procurement strategy with backup sourcing under stochastic supply risk," Complexity, vol. 2019, Article ID 3541352, 15 pages, 2019. 